\title{
Search for new physics with long-lived particles decaying to photons and missing energy in pp collisions at $\sqrt{s}=7 \mathrm{TeV}$
}

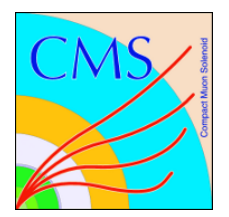

\section{The CMS collaboration}

E-mail: cms-publication-committee-chair@cern.ch

ABSTRACT: A search is performed for long-lived neutral particles decaying into a photon and invisible particles. An example of such a signature is the decay of the lightest neutralino with nonzero lifetime into a gravitino and a photon in gauge-mediated supersymmetry, with the neutralino as the next-to-lightest supersymmetric particle and the gravitino as the lightest. The search uses events containing photons, missing transverse energy, and jets. The impact parameter of the photon relative to the beam-beam collision point can be reconstructed using converted photons. The method is sensitive to lifetimes of the order of 0.1 to $1 \mathrm{~ns}$. The data sample corresponds to an integrated luminosity of $2.23 \mathrm{fb}^{-1}$ in pp collisions at $\sqrt{s}=7 \mathrm{TeV}$, recorded in the first part of 2011 by the CMS experiment at the LHC. Cross-section limits are presented on pair production for such particles, each of which decays into a photon and invisible particles. The observed $95 \%$ confidence level limits vary between 0.11 and $0.21 \mathrm{pb}$, depending on the neutral particle lifetime.

KEYWORDS: Hadron-Hadron Scattering

ArXiv EPRint: 1207.0627 


\section{Contents}

1 Introduction 1

2 Event sample and selections $\quad 2$

3 Photon conversion and the photon impact parameter 4

4 Background determination $\quad 5$

$\begin{array}{lll}5 & \text { Systematic uncertainties } & 7\end{array}$

6 Results and interpretation $\quad 9$

$\begin{array}{lll}7 & \text { Summary } & 9\end{array}$

The CMS collaboration $\quad 13$

\section{Introduction}

New heavy particles with long lifetimes are predicted in many models of physics beyond the standard model (SM), including models with gauge-mediated supersymmetry breaking (GMSB) [1-9] and certain classes of hidden valley models [10]. Such particles may be neutral and decay into photons and invisible particles, which escape detection. Their lifetime is essentially a free parameter of the models, which motivates searches sensitive to a wide range of potential lifetimes. For sufficiently long lifetimes, of order of 1 to $10 \mathrm{~ns}$, measurement of the shower direction by the D0 experiment [11] or time-of-flight by the CDF experiment [12] with an electromagnetic calorimeter can be used to identify such decays, and limits have been obtained by using such techniques at the Tevatron.

In this paper, we present a new analysis method that is sensitive to neutral particles with lifetimes of the order of 0.1 to $1 \mathrm{~ns}$. In a fraction of events, photons undergo conversion into $\mathrm{e}^{+} \mathrm{e}^{-}$pairs in the material of the LHC beam pipe or the CMS charged-particle tracking system, providing a clear experimental signature. The tracks of the electrons can be precisely reconstructed and used to calculate the photon trajectory. In particular, the photon impact parameter with respect to the primary vertex can be determined.

We search for diphoton events with at least one converted photon having a significant impact parameter produced in association with missing transverse energy. Models with gauge-mediated supersymmetry breaking, e.g., general gauge-mediation (GGM), are used to demonstrate the kinematics of the production and decay. Figure 1 shows one of the production and decay diagrams. Assuming that R-parity is conserved [13], SUSY particles are produced in pairs and decay into SM particles and the lightest neutralino $\left(\widetilde{\chi}_{1}^{0}\right)$. The 


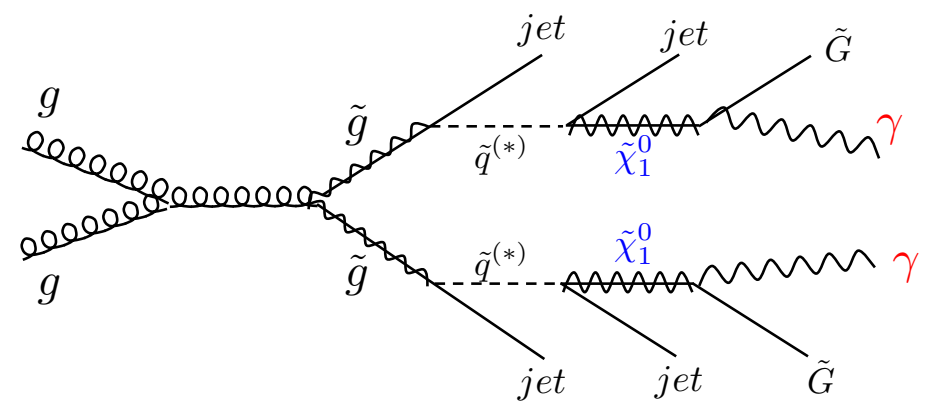

Figure 1. Example diagram of $\widetilde{\chi}_{1}^{0}$ pair production and $\widetilde{\chi}_{1}^{0} \rightarrow \gamma+\widetilde{\mathrm{G}}$ decay.

neutralino decays into a photon and a gravitino $(\widetilde{\mathrm{G}})$, the lightest SUSY particle in this model, which escapes the detector, leading to apparent missing transverse energy $E_{\mathrm{T}}^{\text {miss }}$. Moreover, in many models, the $\widetilde{\chi}_{1}^{0}$ is produced in association with high transverse momentum $\left(p_{\mathrm{T}}\right)$ jets. We consider neutralino proper decay lengths between $c \tau=2$ and $25 \mathrm{~cm}$, corresponding to a lifetime $\tau$ on the order of 0.1 to $1 \mathrm{~ns}$.

The data sample was collected in 2011 by the Compact Muon Solenoid (CMS) experiment at the LHC from proton-proton collisions at a center-of-mass energy of $7 \mathrm{TeV}$, corresponding to an integrated luminosity of $2.23 \pm 0.05 \mathrm{fb}^{-1}$. A detailed description of the CMS detector can be found elsewhere [14]. The detector's central feature is a superconducting solenoid providing a $3.8 \mathrm{~T}$ axial magnetic field along the beam direction. Charged particle trajectories are measured by a silicon pixel and strip tracker system, covering $0 \leq \phi \leq 2 \pi$ in azimuth and $|\eta|<2.5$, where pseudorapidity is defined as $\eta=-\ln \tan \theta / 2$, and $\theta$ is the polar angle with respect to the counterclockwise beam direction. The amount of material crossed by a particle traversing the tracker volume from the interaction region varies from 0.4 to more than 2 radiation lengths, depending on the particle direction. A lead-tungstate crystal electromagnetic calorimeter (ECAL) and a brass-scintillator hadron calorimeter (HCAL) surround the tracker volume. For the barrel and endcap calorimeters $(|\eta|<3.0)$, the modules are arranged in projective towers. Muons are measured with gaseous detectors embedded in the steel return yoke of the magnet. The detector is nearly hermetic with calorimeter coverage up to $|\eta|=5.0$, allowing reliable measurement of $E_{\mathrm{T}}^{\text {miss }}$.

The analysis strategy consists of selecting events with a diphoton final state with at least one converted photon, and then examining the impact parameter of each single photon for a displaced photon signal. Missing transverse energy and at least two jets are also required. The background is estimated using a low $E_{\mathrm{T}}^{\text {miss }}$ control sample. Upper limits on the cross section for pair production of neutral particles, each of which decays into a photon and invisible particles, are then computed as a function of the neutral particle's lifetime.

\section{Event sample and selections}

The data were recorded using the CMS two-level trigger system. This analysis selects events with at least two photons. A diphoton trigger is required with ECAL transverse energy 
thresholds $E_{\mathrm{T}}$ set to values increasing from $32(22)$ to $40 \mathrm{GeV}(28 \mathrm{GeV})$ for the leading (subleading) photon, as the instantaneous luminosity rose over the course of the data-taking period. To ensure being on the plateau of the trigger efficiency, the offline analysis selects events with at least two photons with $E_{\mathrm{T}}>45 \mathrm{GeV}(30 \mathrm{GeV})$ for the leading (sub-leading) photon in the event. The data sample is used both for the selection of signal candidates and for control samples used for background estimation.

The PYтнia 6.4 event generator [15] is used to simulate SUSY signal events. In particular, we generate SUSY GMSB signal datasets in the SPS8 benchmark model [16] with $\Lambda$ around $100 \mathrm{TeV}$. Every event in this sample has two neutralinos, each having a mass of $140 \mathrm{GeV}$. The neutralinos in each dataset have a specific mean lifetime. The signal selection efficiency can depend on the $\widetilde{\chi}_{1}^{0}$ mass, but for masses around $100 \mathrm{GeV}$ this dependence has a negligible effect in this analysis. Each of the neutralinos decays to a photon and a light gravitino. The CMS detector response is fully simulated using GEANT4 [17].

Because of the high luminosity during the run, many events had multiple pp interactions leading to a distribution of primary vertices, called "pile-up". The pile-up conditions at high luminosity affect the event selections, in particular conversion reconstruction efficiencies. The generated pile-up distribution in the Monte Carlo samples was reweighted to reproduce the 2011 data-taking conditions.

The unconverted and converted photon candidates are reconstructed from clusters of energy in the ECAL. At least one photon candidate with transverse energy $E_{\mathrm{T}}>45 \mathrm{GeV}$ and reconstructed in the ECAL barrel region is required. The transverse distribution of energy in the associated cluster of ECAL crystals must be consistent with that expected from a photon, and the energy detected in the HCAL behind the photon shower cannot exceed $5 \%$ of the ECAL energy. To suppress hadronic jets misreconstructed as photon candidates, we require the latter to be isolated from other particles in the tracker, ECAL, and HCAL. A cone of $\Delta R=\sqrt{(\Delta \eta)^{2}+(\Delta \phi)^{2}}=0.4$ is constructed around the candidate's direction, and the scalar sums of transverse energies of tracks and calorimeter deposits within this cone are determined, after excluding the contribution from the candidate itself. The isolation sums are required to be $\sum E_{\mathrm{ECAL}}<0.006 \cdot E_{\mathrm{T}}+4.2 \mathrm{GeV}, \sum E_{\mathrm{HCAL}}<$ $0.0025 \cdot E_{\mathrm{T}}+2.2 \mathrm{GeV}$, and $\sum p_{\mathrm{T}}^{\text {tracks }}<0.001 \cdot E_{\mathrm{T}}+2.0 \mathrm{GeV}$, for the ECAL, HCAL, and tracker, respectively, where $E_{\mathrm{T}}$ is the transverse energy of the photon candidate. To reject electrons, we require that photon candidates do not have associated hit patterns in the pixel detector consistent with an electron track and not be part of a reconstructed conversion track pair.

Photon-like signals that satisfy the same requirements as photons except for isolation are defined as "fake photons". These objects, predominantly jets containing energetic neutral particles such as $\pi^{0} \mathrm{~s}$, are used for the background estimation.

Jets are reconstructed from energy deposits in the calorimeters using the anti- $k_{\mathrm{T}}$ clustering algorithm [18]. The energies of these jets are corrected using the $p_{\mathrm{T}}$ of the charged tracks reconstructed in the tracker [19]. At least two jets are required with $|\eta| \leq 2.6$ and leading jet transverse momentum $p_{\mathrm{T} 1}>80 \mathrm{GeV}$ and sub-leading jet transverse momentum $p_{\mathrm{T} 2}>50 \mathrm{GeV}$.

Missing transverse energy is calculated from calorimeter energy deposits. It is corrected using tracking information for energy missed owing to incomplete calorimeter mea- 

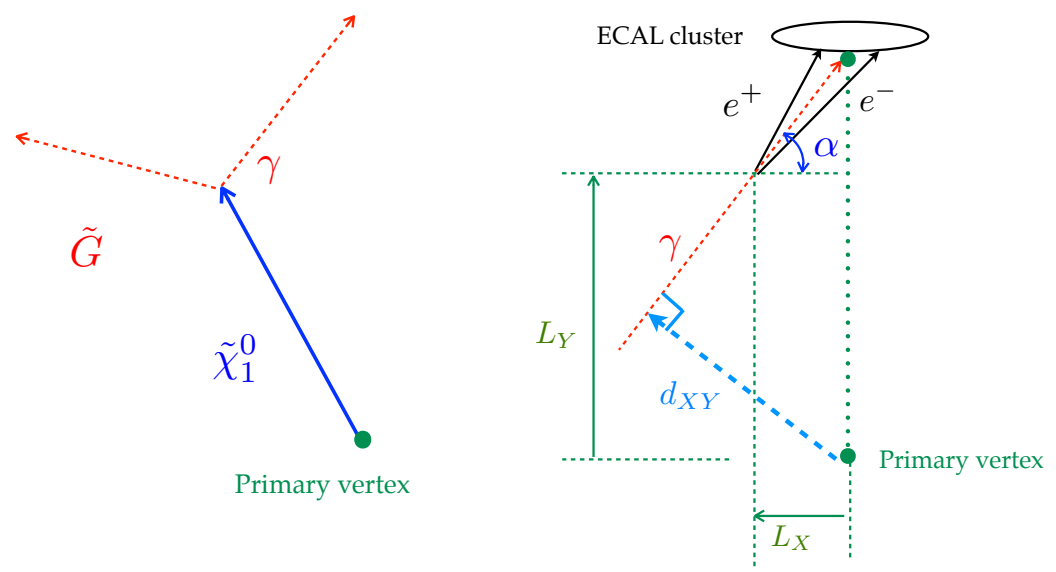

Figure 2. Left: Illustration of $\widetilde{\chi}_{1}^{0} \rightarrow \gamma+\widetilde{\mathrm{G}}$ decay. Right: In $x-y$ plane, a neutralino travels with a finite lifetime and decays into $\gamma+\widetilde{G}$. The diagram shows the photon converting into an $\mathrm{e}^{+} \mathrm{e}^{-}$pair, and the subsequent reconstruction of the impact parameter.

surements of muons and charged hadron energies, especially for soft tracks that do not reach the calorimeters [20]. The $E_{\mathrm{T}}^{\text {miss }}$ threshold of $30 \mathrm{GeV}$ discriminates between signal and background.

\section{Photon conversion and the photon impact parameter}

The CMS silicon tracker provides robust and precise reconstruction of charged-particle momenta in the high-occupancy environment of LHC collisions. Photon conversions in the tracker material can be used to obtain the photon direction. By extrapolation along the momentum direction from the conversion vertex back to the beam line, we can calculate the impact parameter of the displaced photons, as shown in figure 2. If the $\widetilde{\chi}_{1}^{0}$ has a nonzero lifetime, the decay photon can originate from a displaced secondary vertex rather than close to the primary vertex. The momentum direction determined from conversion tracks for this photon will point away from the primary vertex, giving a nonzero impact parameter (IP), which could be a signature for a long-lived $\widetilde{\chi}_{1}^{0}$ signal. The conversion reconstruction efficiency is approximately $5 \%$.

The transverse impact parameter $d_{X Y}$ is the distance of closest approach of the photon trajectory to the beam line in the transverse plane to the beam. The longitudinal impact parameter $d_{Z}$ is the distance of closest approach of the photon trajectory to the primary vertex projected on to the $z$ direction. The photon trajectory is defined as a straight line from the conversion vertex along the conversion momentum. The impact parameters $d_{X Y}$ and $d_{Z}$ are expressed in terms of the vector $\vec{L}=\left(L_{X}, L_{Y}, L_{Z}\right)$ between the conversion point and the primary vertex, and the angle $\alpha$ of the conversion momentum vector $\vec{p}$ projected on to the $x-y$ plane as follows:

$$
\begin{aligned}
d_{X Y} & =-L_{X} \cdot \sin \alpha+L_{Y} \cdot \cos \alpha \\
d_{Z} & =L_{Z}-\frac{L_{X} \cdot p_{X}+L_{Y} \cdot p_{Y}}{p_{\mathrm{T}}} \cdot \frac{p_{Z}}{p_{\mathrm{T}}} .
\end{aligned}
$$


The momentum $\vec{p}$ is calculated as the vector sum of the $\mathrm{e}^{+} \mathrm{e}^{-}$pair momenta at the conversion vertex.

The $\mathrm{e}^{+} \mathrm{e}^{-}$tracks at the conversion vertex are produced with a small opening angle. As a kinematic constraint on their $\theta$ and $\phi$ angular directions, the momenta of the tracks are required to be parallel. This constraint is applied to select conversion tracks. Similarly, the conversion vertex is fitted using kinematic constraints. Three algorithms are used to reconstruct conversions: the "tracker-only" [21, 22], the "ECAL-seeded" [23], and the "Gaussian sum filter" (GSF) [24]. The tracker-only algorithm selects track pairs from all reconstructed tracks, with the kinematic constraint, and fits the conversion vertex; the ECAL-seeded algorithm takes the energy deposits in the ECAL as seeds and then extrapolates back to the tracker to fit the conversion tracks and vertices; the GSF electron algorithm follows a procedure similar to the tracker-only algorithm but uses electrons identified by the GSF algorithm to find the track pairs. The conversions from these three algorithms are merged and the duplicates are removed. Among the reconstructed conversion tracks, the one with the largest number of hits is kept if it passes the necessary quality cut. To be selected, the tracks of two opposite-signed conversion particles both need at least five valid hits; in addition, the conversion vertex requires a valid fit with $\chi^{2}$ probability $>5 \times 10^{-4}$.

In high luminosity conditions, multiple collisions occur within a single bunch crossing, producing a number of primary vertices. The true primary vertex can have a large deviation from the interaction point in the longitudinal direction but a much smaller uncertainty in the transverse direction. To be robust against pile-up conditions, the transverse impact parameter $\left(d_{X Y}\right)$ with respect to the transverse position of the beam line is used in this analysis. To illustrate the $d_{X Y}$ signature, the distribution of its absolute value in data for events with $E_{\mathrm{T}}^{\text {miss }}>30 \mathrm{GeV}$ is compared with a Monte Carlo sample with $\widetilde{\chi}_{1}^{0}$ proper decay length $c \tau=5 \mathrm{~cm}$, as shown in figure 3 . The requirement that $\left|d_{X Y}\right|$ be larger than $0.6 \mathrm{~cm}$ then defines the signal region. This cut on $\left|d_{X Y}\right|$ is derived by optimizing the expected limits on the production cross section. This limit-based optimization is cross-checked against an optimization on the expected signal significance, and the results obtained are the same. In addition, we evaluate the search for several values of the $\left|d_{X Y}\right|$ cut, in the range from 0 to 1.0 $\mathrm{cm}$, and establish that the results are stable for both expected and observed limits. As seen in figure 3, one event passes all selection criteria. The Monte Carlo sample is normalized to the integrated luminosity, and on average 8.3 events from the SPS 8 benchmark model are expected to pass all selections.

The effect of the event selection is illustrated in table 1 for the $c \tau=5 \mathrm{~cm}$ sample. Of the 45057 simulated events, 711 events survive the cuts, for an overall event selection efficiency of $1.58 \%$. Efficiencies for four neutralino lifetimes are given in table 2. On average, the signal efficiency is about $1 \%$ to $2 \%$ after the trigger requirements and selection criteria are applied. Beyond $c \tau=25 \mathrm{~cm}$, the signal efficiency decreases due to the limitations of the tracking algorithms for reconstructing highly displaced tracks.

\section{Background determination}

The background contributions to the sample of events with photon-plus-jets with missing transverse energy arise mainly from QCD events with real photons, and QCD multijet 


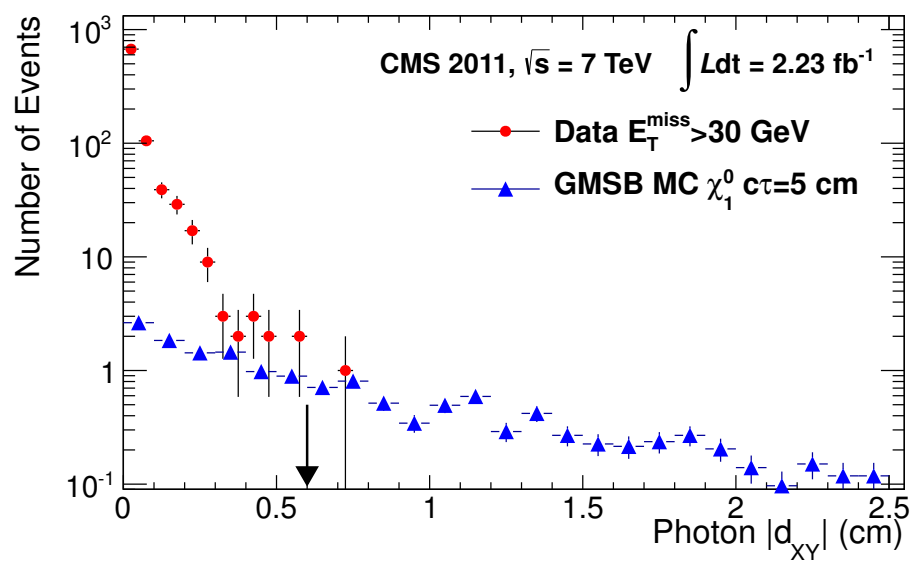

Figure 3. The $\left|d_{X Y}\right|$ distribution for data with $E_{\mathrm{T}}^{\text {miss }}>30 \mathrm{GeV}$ compared with signal simulation for $c \tau=5 \mathrm{~cm}$ normalized to the integrated luminosity of the data. The arrow indicates the $\left|d_{X Y}\right|$ requirement of the signal region.

\begin{tabular}{|l|c|}
\hline Selection & Events in Monte Carlo \\
\hline Total & 45057 \\
\hline Diphoton trigger & 39988 \\
\hline $\begin{array}{l}\text { Leading (sub-leading) photon } E_{\mathrm{T}}>45 \\
(30) \mathrm{GeV}\end{array}$ & 37398 \\
\hline $\begin{array}{l}\text { Any ECAL barrel photon } E_{\mathrm{T}}>45 \mathrm{GeV} \\
\text { and photon identification }\end{array}$ & 27766 \\
\hline Jets $p_{\mathrm{T} 1}>80 \mathrm{GeV}$ and $p_{\mathrm{T} 2}>50 \mathrm{GeV}$ & 26229 \\
\hline Conversion selection & 1602 \\
\hline$E_{\mathrm{T}}^{\text {miss }}>30 \mathrm{GeV}$ & 1542 \\
\hline$\left|d_{X Y}\right|>0.6 \mathrm{~cm}$ & 711 \\
\hline
\end{tabular}

Table 1. Signal selection cut flow for $\mathrm{c} \tau=5 \mathrm{~cm}$.

\begin{tabular}{|l|cccc|}
\hline$c \tau[\mathrm{cm}]$ & 2 & 5 & 10 & 25 \\
\hline Efficiency & $0.92 \%$ & $1.58 \%$ & $1.80 \%$ & $1.39 \%$ \\
\hline Statistical errors & $0.05 \%$ & $0.06 \%$ & $0.06 \%$ & $0.06 \%$ \\
\hline
\end{tabular}

Table 2. Event selection efficiency vs. $\widetilde{\chi}_{1}^{0}$ proper decay length.

events, due to the effect of finite detector resolution on the measurement of $E_{\mathrm{T}}^{\text {miss }}$. In QCD events with real photons, there are typically one real photon and one jet that is misidentified as a photon in the final selection. The rate of QCD multijet events is sufficiently large to have a finite probability for jets to be misidentified as photons. There are additional sources of background with intrinsic missing transverse energy, such as the $\mathrm{W}+$ jets process, but these are heavily suppressed by the $E_{\mathrm{T}}$ cut for the photon and make a negligible contribution to the final selection. The $\widetilde{\chi}_{1}^{0} \rightarrow \gamma+\widetilde{\mathrm{G}}$ decay has two signatures: $E_{\mathrm{T}}^{\text {miss }}$ from the unseen $\widetilde{\mathrm{G}}$ and a large impact parameter from the displaced photons. The strategy for 
determining the background is to use control samples that are kinematically similar to the candidate sample while having no real $E_{\mathrm{T}}^{\text {miss }}$. The data with $E_{\mathrm{T}}^{\text {miss }}<20 \mathrm{GeV}$ are selected as such a control sample. The background is then estimated as the number of events with $E_{\mathrm{T}}^{\text {miss }}<20 \mathrm{GeV}$ and satisfying all the other selection criteria.

To ensure that the $E_{\mathrm{T}}^{\text {miss }}<20 \mathrm{GeV}$ region of data gives a correct estimation of the background, the $\left|d_{X Y}\right|$ distributions of fake photons, defined previously, and isolated photons are compared. First, the $\left|d_{X Y}\right|$ distribution of fake photons for events with $E_{\mathrm{T}}^{\text {miss }}<20 \mathrm{GeV}$ (background region) is normalized by the total number of conversions and compared with the $\left|d_{X Y}\right|$ distribution of fake photons in the region where $E_{\mathrm{T}}^{\text {miss }}>30 \mathrm{GeV}$ (signal region), as shown in figure 4 (top). The $\left|d_{X Y}\right|$ distribution in the $E_{\mathrm{T}}^{\text {miss }}<20 \mathrm{GeV}$ (background) region is reweighted by the conversion vertex $\chi^{2}$ probability of the $E_{\mathrm{T}}^{\mathrm{miss}}<20 \mathrm{GeV}$ (signal) region. This takes into account that the distributions of the $\chi^{2}$ probability are not the same in the background and signal region. Second, the $\left|d_{X Y}\right|$ distributions of isolated photons and fake photons in the low $E_{\mathrm{T}}^{\mathrm{miss}}$ region are compared by normalizing to the total number of conversions, also reweighted by the conversion vertex $\chi^{2}$ probability, as shown in figure 4(bottom). The $\left|d_{X Y}\right|$ distributions of fake photons and isolated photons agree, and thus we conclude that the $\left|d_{X Y}\right|$ distribution for events with $E_{\mathrm{T}}^{\text {miss }}<20 \mathrm{GeV}$ gives a good description of the background. The residual differences in the fake and isolated photon distributions in the low $E_{\mathrm{T}}^{\text {miss }}$ control sample are consistent with the expected enhancement of jets misreconstructed as photons in the fake photon sample. We therefore take the isolated photon sample in the low $E_{\mathrm{T}}^{\text {miss }}$ control sample as the basis for the background estimate in the signal region, after we apply conversion vertex $\chi^{2}$ probability reweighting.

The isolated photon $\left|d_{X Y}\right|$ distributions for the signal and background regions are shown in figure 5. They are normalized to the same number of conversions and reweighted by the conversion vertex $\chi^{2}$ probability to predict the number of background events. A requirement that $\left|d_{X Y}\right|>0.6 \mathrm{~cm}$ is applied to the background region $E_{\mathrm{T}}^{\text {miss }}<20 \mathrm{GeV}$, which gives a total background of $0.78_{-0.48}^{+1.25}$ events. As a cross check, Monte Carlo background samples are fitted with a general functional form to extrapolate the expected tail of events for isolated photons beyond the $\left|d_{X Y}\right|>0.6 \mathrm{~cm}$ cut. Applying this general functional form to the background sample derived from data and integrating the expected rate above the $\left|d_{X Y}\right|>0.6 \mathrm{~cm}$ cut gives a background estimate consistent with the number quoted above.

\section{$5 \quad$ Systematic uncertainties}

Table 3 summarizes the systematic uncertainties affecting this analysis. To determine the uncertainty in the conversion reconstruction efficiency, we compared the predicted and observed numbers of conversions for $Z \rightarrow \mu \mu \gamma$ events. For photons with $E_{\mathrm{T}}>20 \mathrm{GeV}$, a scale factor of $0.87 \pm 0.08$ is derived. High $E_{\mathrm{T}}$ photons have a reconstruction efficiency of $(7.0 \pm 0.5) \%$ in the Drell-Yan to dimuon Monte Carlo samples, and the corresponding high $E_{\mathrm{T}}$ photon efficiency in the GMSB Monte Carlo samples is $(6.0 \pm 0.7) \%$, yielding a $\mathrm{Z} \rightarrow \mu \mu \gamma$-GMSB scale factor of $1.16 \pm 0.08$. Considering the correlations between the conversion reconstruction efficiency and the high- $E_{\mathrm{T}}$ dependence, the uncertainty of conversion reconstruction is determined to be $21 \%$ for the signal region. 

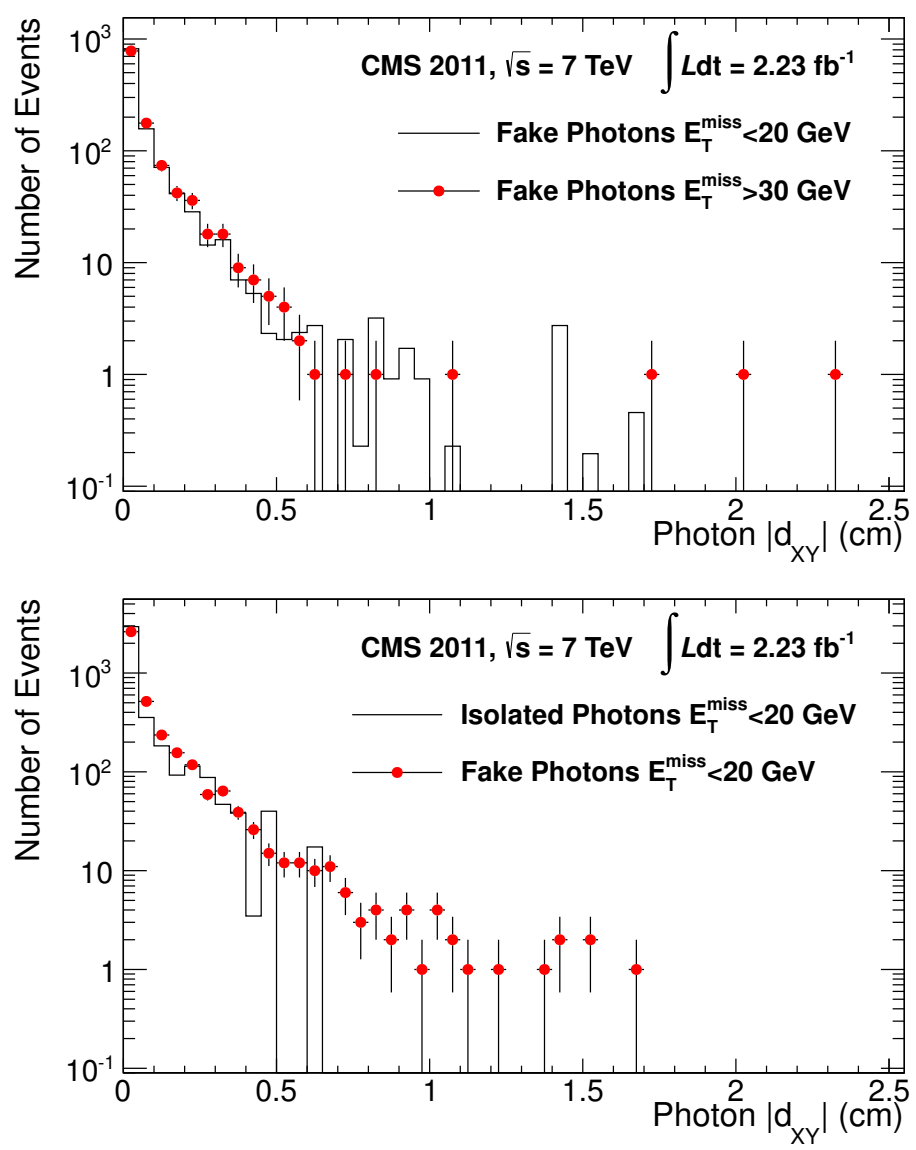

Figure 4. Top: fake photon $\left|d_{X Y}\right|$ distributions in the background $\left(E_{\mathrm{T}}^{\text {miss }}<20 \mathrm{GeV}\right)$ and signal $\left(E_{\mathrm{T}}^{\text {miss }}>30 \mathrm{GeV}\right)$ regions. Bottom: isolated and fake photon $\left|d_{X Y}\right|$ distributions in the background region $\left(E_{\mathrm{T}}^{\text {miss }}<20 \mathrm{GeV}\right)$.

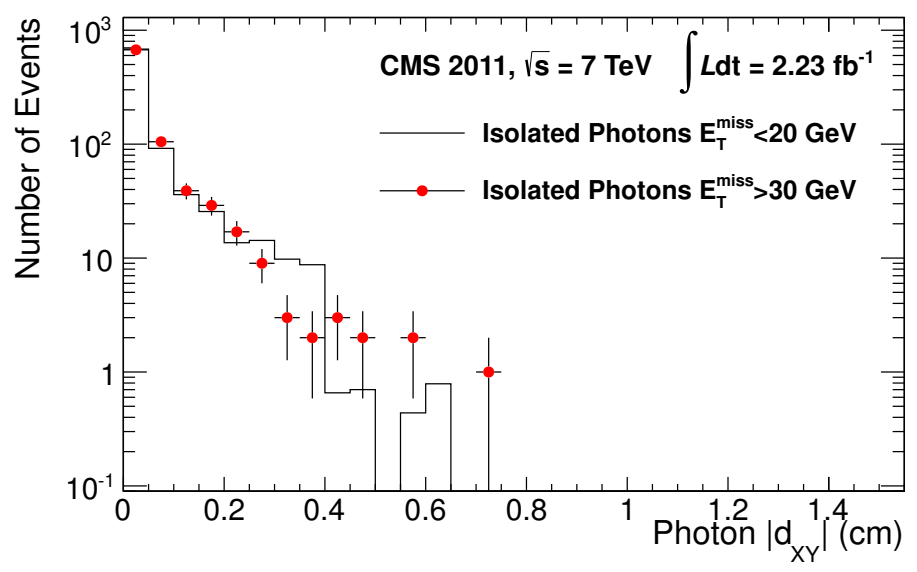

Figure 5. $\left|d_{X Y}\right|$ distributions for isolated photons: background region $\left(E_{\mathrm{T}}^{\text {miss }}<20 \mathrm{GeV}\right)$ compared to the signal region $\left(E_{\mathrm{T}}^{\text {miss }}>30 \mathrm{GeV}\right)$. 


\begin{tabular}{|l|c|}
\hline Systematics & Uncertainty (\%) \\
\hline Integrated luminosity & 2.2 \\
Jet $p_{\mathrm{T}} / E_{\mathrm{T}}^{\text {miss }}$ energy scale & $<0.5$ \\
Pile-up & 2.5 \\
Photon identification data/MC scale & 2.6 \\
Photon-electron difference & 0.5 \\
Conversion reconstruction efficiency & 21 \\
Photon $d_{X Y}$ resolution & $<0.5$ \\
Total & 25 \\
\hline
\end{tabular}

Table 3. Summary of systematic uncertainties.

The $d_{X Y}$ resolution is studied by comparing the $d_{X Y}$ resolution for converted photons from $\mathrm{Z} \rightarrow \mu \mu \gamma$ events between Monte Carlo and data. The data and Monte Carlo samples are in agreement and a $d_{X Y}$ resolution of $0.06 \mathrm{~cm}$ extracted from data gives less than $0.5 \%$ uncertainty for the signal selection. The resolution uncertainty includes tails in the fitted resolution function.

Other sources of uncertainty include the integrated luminosity $(2.2 \%)$, the jet $p_{\mathrm{T}} / E_{\mathrm{T}}^{\text {miss }}$ selection requirement, and photon identification. The jet $p_{\mathrm{T}} / E_{\mathrm{T}}^{\text {miss }}$ resolution contributes less than $0.5 \%$ to the total uncertainty $[19,20]$. The photon identification efficiency is studied in [25] and includes the uncertainties from pile-up (2.5\%), the photon data/MC scale measured in a $\mathrm{Z} \rightarrow$ ee data sample (2.6\%), and the photon-electron identification difference as studied in $\mathrm{MC}$ samples $(0.5 \%)$. Other sources of uncertainty give negligible contributions to the systematic uncertainty. The systematic error from the $d_{X Y}$ cut is taken into account when evaluating the limits on the contribution from the photon $d_{X Y}$ resolution. The total systematic uncertainty is determined to be $25 \%$ by considering all the correlations among pile-up, photon identification data/MC scale, and conversion reconstruction efficiency.

\section{Results and interpretation}

One event with $\left|d_{X Y}\right|=0.74 \mathrm{~cm}$ and $E_{\mathrm{T}}^{\text {miss }}=44.9 \mathrm{GeV}$ satisfying all the other selection criteria is observed. The estimated background is $0.78_{-0.48}^{+1.25}$ events. We determine the upper limits for the cross section for pair production of neutral particles, each of which decays into one photon and invisible particles. A CLs limit setting method [26, 27] is employed using log-normal uncertainties for the total background rate to incorporate the uncertainties in the total background rate, integrated luminosity, and total acceptance times efficiency. The observed $95 \%$ confidence level limits vary between 0.11 and $0.21 \mathrm{pb}$, depending on the neutral particle proper decay length (table 4 and figure 6 ).

\section{Summary}

We have introduced a novel method using the photon conversion impact parameter to search for new physics involving long-lived particles decaying into photons. The high resolution 


\begin{tabular}{|c|cccc|}
\hline$c \tau(\mathrm{cm})$ & 2 & 5 & 10 & 25 \\
\hline$\sigma(\mathrm{pb}) 95 \% \mathrm{CL}$ & 0.21 & 0.12 & 0.11 & 0.14 \\
\hline
\end{tabular}

Table 4. 95\% confidence level (CL) upper limits on the cross section for pair production of neutral particles, each of which decays into a photon and invisible particles, as a function of the neutral particle proper decay length.

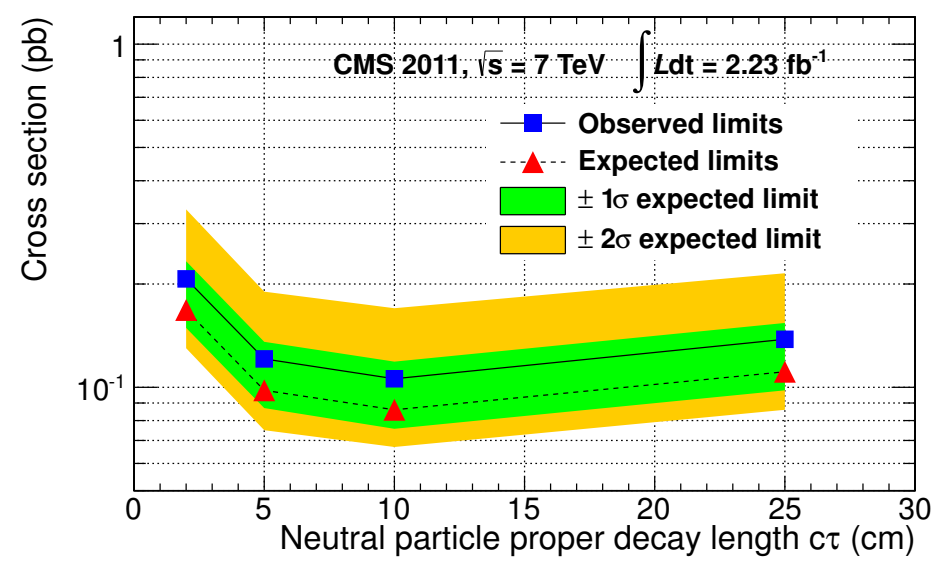

Figure 6. $95 \%$ confidence level upper limits on the pair production cross section for neutral particles, each of which decays into a photon and invisible particles, as a function of the neutral particle proper decay length. The observed values as a function of the neutral particle proper decay length are shown by the solid line. The dashed line indicates the expected median of results for the background-only hypothesis, while the green (dark) and yellow (light) bands indicate the ranges that are expected to contain $68 \%$ and $95 \%$ of all observed excursions from the median, respectively.

of the impact parameter reconstruction enabled our analysis to probe for new physics to much lower values of $E_{\mathrm{T}}^{\text {miss }}$ compared to other LHC searches [25]. The search was performed using the final state of photons, jets, and missing transverse energy. Cross-section limits on pair production for such particles, each of which decays into a photon and invisible particles, were set as a function of the long-lived particle's lifetime. These upper limits are applicable to a general class of new physics processes with diphotons in the final state.

\section{Acknowledgments}

We congratulate our colleagues in the CERN accelerator departments for the excellent performance of the LHC machine. We thank the technical and administrative staff at CERN and other CMS institutes, and acknowledge support from: FMSR (Austria); FNRS and FWO (Belgium); CNPq, CAPES, FAPERJ, and FAPESP (Brazil); MES (Bulgaria); CERN; CAS, MoST, and NSFC (China); COLCIENCIAS (Colombia); MSES (Croatia); RPF (Cyprus); MoER, SF0690030s09 and ERDF (Estonia); Academy of Finland, MEC, and HIP (Finland); CEA and CNRS/IN2P3 (France); BMBF, DFG, and HGF (Germany); GSRT (Greece); OTKA and NKTH (Hungary); DAE and DST (India); IPM (Iran); SFI (Ireland); INFN (Italy); NRF and WCU (Korea); LAS (Lithuania); CINVESTAV, CONA- 
CYT, SEP, and UASLP-FAI (Mexico); MSI (New Zealand); PAEC (Pakistan); MSHE and NSC (Poland); FCT (Portugal); JINR (Armenia, Belarus, Georgia, Ukraine, Uzbekistan); MON, RosAtom, RAS and RFBR (Russia); MSTD (Serbia); MICINN and CPAN (Spain); Swiss Funding Agencies (Switzerland); NSC (Taipei); TUBITAK and TAEK (Turkey); STFC (United Kingdom); DOE and NSF (USA).

Open Access. This article is distributed under the terms of the Creative Commons Attribution License which permits any use, distribution and reproduction in any medium, provided the original author(s) and source are credited.

\section{References}

[1] S. Ambrosanio, G.L. Kane, G.D. Kribs, S.P. Martin and S. Mrenna, Search for supersymmetry with a light gravitino at the Fermilab Tevatron and CERN LEP colliders, Phys. Rev. D 54 (1996) 5395 [hep-ph/9605398] [INSPIRE].

[2] S. Dimopoulos, S.D. Thomas and J.D. Wells, Sparticle spectroscopy and electroweak symmetry breaking with gauge mediated supersymmetry breaking, Nucl. Phys. B 488 (1997) 39 [hep-ph/9609434] [INSPIRE].

[3] G. Giudice and R. Rattazzi, Theories with gauge mediated supersymmetry breaking, Phys. Rept. 322 (1999) 419 [hep-ph/9801271] [INSPIRE].

[4] L.M. Carpenter, Surveying the phenomenology of general gauge mediation, arXiv:0812.2051 [INSPIRE].

[5] A. Rajaraman, Y. Shirman, J. Smidt and F. Yu, Parameter space of general gauge mediation, Phys. Lett. B 678 (2009) 367 [arXiv: 0903. 0668] [INSPIRE].

[6] S. Abel, M.J. Dolan, J. Jaeckel and V.V. Khoze, Phenomenology of pure general gauge mediation, JHEP 12 (2009) 001 [arXiv:0910.2674] [INSPIRE].

[7] P. Meade, M. Reece and D. Shih, Prompt decays of general neutralino NLSPs at the Tevatron, JHEP 05 (2010) 105 [arXiv:0911.4130] [INSPIRE].

[8] P. Meade, M. Reece and D. Shih, Long-lived neutralino NLSPs, JHEP 10 (2010) 067 [arXiv: 1006.4575] [INSPIRE].

[9] J.T. Ruderman and D. Shih, General neutralino NLSPs at the early LHC, JHEP 08 (2012) 159 [arXiv: 1103.6083] [INSPIRE].

[10] M.J. Strassler and K.M. Zurek, Echoes of a hidden valley at hadron colliders, Phys. Lett. B 651 (2007) 374 [hep-ph/0604261] [INSPIRE].

[11] D0 collaboration, V. Abazov et al., Search for long-lived particles decaying into electron or photon pairs with the D0 detector, Phys. Rev. Lett. 101 (2008) 111802 [arXiv:0806.2223] [INSPIRE].

[12] CDF collaboration, A. Abulencia et al., Search for heavy, long-lived particles that decay to photons at CDF II, Phys. Rev. Lett. 99 (2007) 121801 [arXiv:0704.0760] [INSPIRE].

[13] G.R. Farrar and P. Fayet, Phenomenology of the production, decay and detection of new hadronic states associated with supersymmetry, Phys. Lett. B 76 (1978) 575 [INSPIRE].

[14] CMS collaboration, The CMS experiment at the CERN LHC, 2008 JINST 3 S08004 [INSPIRE]. 
[15] T. Sjöstrand, S. Mrenna and P.Z. Skands, PYTHIA 6.4 physics and manual, JHEP 05 (2006) 026 [hep-ph/0603175] [INSPIRE].

[16] B. Allanach et al., The Snowmass points and slopes: benchmarks for SUSY searches, Eur. Phys. J. C 25 (2002) 113 [hep-ph/0202233] [INSPIRE].

[17] GEANT4 collaboration, S. Agostinelli et al., GEANT4: a simulation toolkit, Nucl. Instrum. Meth. A 506 (2003) 250 [INSPIRE].

[18] M. Cacciari, G.P. Salam and G. Soyez, The anti- $k_{t}$ jet clustering algorithm, JHEP 04 (2008) 063 [arXiv: 0802.1189] [INSPIRE].

[19] CMS collaboration, Jet performance in pp collisions at $7 \mathrm{TeV}$, PAS-JME-10-003 (Jet Performance in pp Collisions at $7 \mathrm{TeV}$ ).

[20] CMS collaboration, Missing transverse energy performance of the CMS detector, 2011 JINST 6 P09001.

[21] CMS collaboration, V. Khachatryan et al., CMS tracking performance results from early LHC operation, Eur. Phys. J. C 70 (2010) 1165 [arXiv: 1007.1988] [INSPIRE].

[22] CMS collaboration, Studies of tracker material, PAS-TRK-10-003 (2010).

[23] N. Marinelli, Track finding and identification of converted photons, CMS-NOTE-2006-005 (2006).

[24] CMS collaboration, Electromagnetic physics objects commissioning with first LHC data, PAS-EGM-10-001 (Electromagnetic physics objects commissioning with first LHC data).

[25] CMS collaboration, S. Chatrchyan et al., Search for supersymmetry in pp collisions at $\sqrt{s}=7 \mathrm{TeV}$ in events with two photons and missing transverse energy, Phys. Rev. Lett. 106 (2011) 211802 [arXiv:1103.0953] [INSPIRE].

[26] A.L. Read, Presentation of search results: the CL(s) technique, J. Phys. G 28 (2002) 2693 [INSPIRE].

[27] L. Moneta et al., The RooStats Project, PoS (ACAT2010) 057 [arXiv:1009.1003] [INSPIRE]. 


\section{The CMS collaboration}

\section{Yerevan Physics Institute, Yerevan, Armenia}

S. Chatrchyan, V. Khachatryan, A.M. Sirunyan, A. Tumasyan

\section{Institut für Hochenergiephysik der OeAW, Wien, Austria}

W. Adam, E. Aguilo, T. Bergauer, M. Dragicevic, J. Erö, C. Fabjan ${ }^{1}$, M. Friedl, R. Frühwirth ${ }^{1}$, V.M. Ghete, J. Hammer, N. Hörmann, J. Hrubec, M. Jeitler ${ }^{1}$, W. Kiesenhofer, V. Knünz, M. Krammer ${ }^{1}$, I. Krätschmer, D. Liko, I. Mikulec, M. Pernicka ${ }^{\dagger}$, B. Rahbaran, C. Rohringer, H. Rohringer, R. Schöfbeck, J. Strauss, A. Taurok, W. Waltenberger, G. Walzel, E. Widl, C.-E. Wulz ${ }^{1}$

National Centre for Particle and High Energy Physics, Minsk, Belarus

V. Mossolov, N. Shumeiko, J. Suarez Gonzalez

\section{Universiteit Antwerpen, Antwerpen, Belgium}

M. Bansal, S. Bansal, T. Cornelis, E.A. De Wolf, X. Janssen, S. Luyckx, L. Mucibello, S. Ochesanu, B. Roland, R. Rougny, M. Selvaggi, Z. Staykova, H. Van Haevermaet, P. Van Mechelen, N. Van Remortel, A. Van Spilbeeck

\section{Vrije Universiteit Brussel, Brussel, Belgium}

F. Blekman, S. Blyweert, J. D'Hondt, R. Gonzalez Suarez, A. Kalogeropoulos, M. Maes, A. Olbrechts, W. Van Doninck, P. Van Mulders, G.P. Van Onsem, I. Villella

\section{Université Libre de Bruxelles, Bruxelles, Belgium}

B. Clerbaux, G. De Lentdecker, V. Dero, A.P.R. Gay, T. Hreus, A. Léonard, P.E. Marage,

T. Reis, L. Thomas, G. Vander Marcken, C. Vander Velde, P. Vanlaer, J. Wang

\section{Ghent University, Ghent, Belgium}

V. Adler, K. Beernaert, A. Cimmino, S. Costantini, G. Garcia, M. Grunewald, B. Klein, J. Lellouch, A. Marinov, J. Mccartin, A.A. Ocampo Rios, D. Ryckbosch, N. Strobbe, F. Thyssen, M. Tytgat, P. Verwilligen, S. Walsh, E. Yazgan, N. Zaganidis

Université Catholique de Louvain, Louvain-la-Neuve, Belgium

S. Basegmez, G. Bruno, R. Castello, L. Ceard, C. Delaere, T. du Pree, D. Favart, L. Forthomme, A. Giammanco ${ }^{2}$, J. Hollar, V. Lemaitre, J. Liao, O. Militaru, C. Nuttens, D. Pagano, A. Pin, K. Piotrzkowski, N. Schul, J.M. Vizan Garcia

Université de Mons, Mons, Belgium

N. Beliy, T. Caebergs, E. Daubie, G.H. Hammad

\section{Centro Brasileiro de Pesquisas Fisicas, Rio de Janeiro, Brazil}

G.A. Alves, M. Correa Martins Junior, D. De Jesus Damiao, T. Martins, M.E. Pol, M.H.G. Souza

\section{Universidade do Estado do Rio de Janeiro, Rio de Janeiro, Brazil}

W.L. Aldá Júnior, W. Carvalho, A. Custódio, E.M. Da Costa, C. De Oliveira Martins, S. Fonseca De Souza, D. Matos Figueiredo, L. Mundim, H. Nogima, V. Oguri, W.L. Prado Da Silva, A. Santoro, L. Soares Jorge, A. Sznajder 
Instituto de Fisica Teorica, Universidade Estadual Paulista, Sao Paulo, Brazil T.S. Anjos $^{3}$, C.A. Bernardes ${ }^{3}$, F.A. Dias ${ }^{4}$, T.R. Fernandez Perez Tomei, E. M. Gregores ${ }^{3}$, C. Lagana, F. Marinho, P.G. Mercadante ${ }^{3}$, S.F. Novaes, Sandra S. Padula

Institute for Nuclear Research and Nuclear Energy, Sofia, Bulgaria

V. Genchev ${ }^{5}$, P. Iaydjiev ${ }^{5}$, S. Piperov, M. Rodozov, S. Stoykova, G. Sultanov, V. Tcholakov,

R. Trayanov, M. Vutova

University of Sofia, Sofia, Bulgaria

A. Dimitrov, R. Hadjiiska, V. Kozhuharov, L. Litov, B. Pavlov, P. Petkov

Institute of High Energy Physics, Beijing, China

J.G. Bian, G.M. Chen, H.S. Chen, C.H. Jiang, D. Liang, S. Liang, X. Meng, J. Tao, J. Wang, X. Wang, Z. Wang, H. Xiao, M. Xu, J. Zang, Z. Zhang

State Key Lab. of Nucl. Phys. and Tech., Peking University, Beijing, China

C. Asawatangtrakuldee, Y. Ban, Y. Guo, W. Li, S. Liu, Y. Mao, S.J. Qian, H. Teng,

D. Wang, L. Zhang, W. Zou

Universidad de Los Andes, Bogota, Colombia

C. Avila, J.P. Gomez, B. Gomez Moreno, A.F. Osorio Oliveros, J.C. Sanabria

Technical University of Split, Split, Croatia

N. Godinovic, D. Lelas, R. Plestina ${ }^{6}$, D. Polic, I. Puljak ${ }^{5}$

University of Split, Split, Croatia

Z. Antunovic, M. Kovac

Institute Rudjer Boskovic, Zagreb, Croatia

V. Brigljevic, S. Duric, K. Kadija, J. Luetic, S. Morovic

University of Cyprus, Nicosia, Cyprus

A. Attikis, M. Galanti, G. Mavromanolakis, J. Mousa, C. Nicolaou, F. Ptochos, P.A. Razis

Charles University, Prague, Czech Republic

M. Finger, M. Finger Jr.

Academy of Scientific Research and Technology of the Arab Republic of Egypt, Egyptian Network of High Energy Physics, Cairo, Egypt

Y. Assran ${ }^{7}$, S. Elgammal ${ }^{8}$, A. Ellithi Kamel ${ }^{9}$, S. Khalil ${ }^{8}$, M.A. Mahmoud ${ }^{10}$, A. Radi ${ }^{11,12}$

National Institute of Chemical Physics and Biophysics, Tallinn, Estonia

M. Kadastik, M. Müntel, M. Raidal, L. Rebane, A. Tiko

Department of Physics, University of Helsinki, Helsinki, Finland

P. Eerola, G. Fedi, M. Voutilainen

Helsinki Institute of Physics, Helsinki, Finland

J. Härkönen, A. Heikkinen, V. Karimäki, R. Kinnunen, M.J. Kortelainen, T. Lampén,

K. Lassila-Perini, S. Lehti, T. Lindén, P. Luukka, T. Mäenpää, T. Peltola, E. Tuominen,

J. Tuominiemi, E. Tuovinen, D. Ungaro, L. Wendland 
Lappeenranta University of Technology, Lappeenranta, Finland

K. Banzuzi, A. Karjalainen, A. Korpela, T. Tuuva

DSM/IRFU, CEA/Saclay, Gif-sur-Yvette, France

M. Besancon, S. Choudhury, M. Dejardin, D. Denegri, B. Fabbro, J.L. Faure, F. Ferri, S. Ganjour, A. Givernaud, P. Gras, G. Hamel de Monchenault, P. Jarry, E. Locci, J. Malcles, L. Millischer, A. Nayak, J. Rander, A. Rosowsky, I. Shreyber, M. Titov

Laboratoire Leprince-Ringuet, Ecole Polytechnique, IN2P3-CNRS, Palaiseau, France

S. Baffioni, F. Beaudette, L. Benhabib, L. Bianchini, M. Bluj ${ }^{13}$, C. Broutin, P. Busson, C. Charlot, N. Daci, T. Dahms, L. Dobrzynski, R. Granier de Cassagnac, M. Haguenauer, P. Miné, C. Mironov, I.N. Naranjo, M. Nguyen, C. Ochando, P. Paganini, D. Sabes, R. Salerno, Y. Sirois, C. Veelken, A. Zabi

Institut Pluridisciplinaire Hubert Curien, Université de Strasbourg, Université de Haute Alsace Mulhouse, CNRS/IN2P3, Strasbourg, France

J.-L. Agram ${ }^{14}$, J. Andrea, D. Bloch, D. Bodin, J.-M. Brom, M. Cardaci, E.C. Chabert, C. Collard, E. Conte ${ }^{14}$, F. Drouhin ${ }^{14}$, C. Ferro, J.-C. Fontaine ${ }^{14}$, D. Gelé, U. Goerlach, P. Juillot, A.-C. Le Bihan, P. Van Hove

Centre de Calcul de l'Institut National de Physique Nucleaire et de Physique des Particules (IN2P3), Villeurbanne, France

F. Fassi, D. Mercier

Université de Lyon, Université Claude Bernard Lyon 1, CNRS-IN2P3, Institut de Physique Nucléaire de Lyon, Villeurbanne, France

S. Beauceron, N. Beaupere, O. Bondu, G. Boudoul, J. Chasserat, R. Chierici ${ }^{5}$, D. Contardo, P. Depasse, H. El Mamouni, J. Fay, S. Gascon, M. Gouzevitch, B. Ille, T. Kurca, M. Lethuillier, L. Mirabito, S. Perries, V. Sordini, Y. Tschudi, P. Verdier, S. Viret

Institute of High Energy Physics and Informatization, Tbilisi State University, Tbilisi, Georgia

Z. Tsamalaidze ${ }^{15}$

RWTH Aachen University, I. Physikalisches Institut, Aachen, Germany

G. Anagnostou, S. Beranek, M. Edelhoff, L. Feld, N. Heracleous, O. Hindrichs, R. Jussen, K. Klein, J. Merz, A. Ostapchuk, A. Perieanu, F. Raupach, J. Sammet, S. Schael, D. Sprenger, H. Weber, B. Wittmer, V. Zhukov ${ }^{16}$

RWTH Aachen University, III. Physikalisches Institut A, Aachen, Germany M. Ata, J. Caudron, E. Dietz-Laursonn, D. Duchardt, M. Erdmann, R. Fischer, A. Güth, T. Hebbeker, C. Heidemann, K. Hoepfner, D. Klingebiel, P. Kreuzer, C. Magass, M. Merschmeyer, A. Meyer, M. Olschewski, P. Papacz, H. Pieta, H. Reithler, S.A. Schmitz, L. Sonnenschein, J. Steggemann, D. Teyssier, M. Weber 
RWTH Aachen University, III. Physikalisches Institut B, Aachen, Germany M. Bontenackels, V. Cherepanov, Y. Erdogan, G. Flügge, H. Geenen, M. Geisler, W. Haj Ahmad, F. Hoehle, B. Kargoll, T. Kress, Y. Kuessel, A. Nowack, L. Perchalla, O. Pooth, P. Sauerland, A. Stahl

\section{Deutsches Elektronen-Synchrotron, Hamburg, Germany}

M. Aldaya Martin, J. Behr, W. Behrenhoff, U. Behrens, M. Bergholz ${ }^{17}$, A. Bethani, K. Borras, A. Burgmeier, A. Cakir, L. Calligaris, A. Campbell, E. Castro, F. Costanza, D. Dammann, C. Diez Pardos, G. Eckerlin, D. Eckstein, G. Flucke, A. Geiser, I. Glushkov, P. Gunnellini, S. Habib, J. Hauk, G. Hellwig, H. Jung, M. Kasemann, P. Katsas, C. Kleinwort, H. Kluge, A. Knutsson, M. Krämer, D. Krücker, E. Kuznetsova, W. Lange, W. Lohmann ${ }^{17}$, B. Lutz, R. Mankel, I. Marfin, M. Marienfeld, I.-A. MelzerPellmann, A.B. Meyer, J. Mnich, A. Mussgiller, S. Naumann-Emme, J. Olzem, H. Perrey, A. Petrukhin, D. Pitzl, A. Raspereza, P.M. Ribeiro Cipriano, C. Riedl, E. Ron, M. Rosin, J. Salfeld-Nebgen, R. Schmidt ${ }^{17}$, T. Schoerner-Sadenius, N. Sen, A. Spiridonov, M. Stein, R. Walsh, C. Wissing

\section{University of Hamburg, Hamburg, Germany}

C. Autermann, V. Blobel, J. Draeger, H. Enderle, J. Erfle, U. Gebbert, M. Görner, T. Hermanns, R.S. Höing, K. Kaschube, G. Kaussen, H. Kirschenmann, R. Klanner, J. Lange, B. Mura, F. Nowak, T. Peiffer, N. Pietsch, D. Rathjens, C. Sander, H. Schettler, P. Schleper, E. Schlieckau, A. Schmidt, M. Schröder, T. Schum, M. Seidel, V. Sola, H. Stadie, G. Steinbrück, J. Thomsen, L. Vanelderen

Institut für Experimentelle Kernphysik, Karlsruhe, Germany

C. Barth, J. Berger, C. Böser, T. Chwalek, W. De Boer, A. Descroix, A. Dierlamm, M. Feindt, M. Guthoff ${ }^{5}$, C. Hackstein, F. Hartmann, T. Hauth ${ }^{5}$, M. Heinrich, H. Held, K.H. Hoffmann, S. Honc, I. Katkov ${ }^{16}$, J.R. Komaragiri, P. Lobelle Pardo, D. Martschei, S. Mueller, Th. Müller, M. Niegel, A. Nürnberg, O. Oberst, A. Oehler, J. Ott, G. Quast, K. Rabbertz, F. Ratnikov, N. Ratnikova, S. Röcker, A. Scheurer, F.-P. Schilling, G. Schott, H.J. Simonis, F.M. Stober, D. Troendle, R. Ulrich, J. Wagner-Kuhr, S. Wayand, T. Weiler, M. Zeise

Institute of Nuclear Physics "Demokritos", Aghia Paraskevi, Greece

G. Daskalakis, T. Geralis, S. Kesisoglou, A. Kyriakis, D. Loukas, I. Manolakos, A. Markou, C. Markou, C. Mavrommatis, E. Ntomari

University of Athens, Athens, Greece

L. Gouskos, T.J. Mertzimekis, A. Panagiotou, N. Saoulidou

University of Ioánnina, Ioánnina, Greece

I. Evangelou, C. Foudas, P. Kokkas, N. Manthos, I. Papadopoulos, V. Patras

KFKI Research Institute for Particle and Nuclear Physics, Budapest, Hungary G. Bencze, C. Hajdu, P. Hidas, D. Horvath ${ }^{18}$, F. Sikler, V. Veszpremi, G. Vesztergombi ${ }^{19}$

Institute of Nuclear Research ATOMKI, Debrecen, Hungary

N. Beni, S. Czellar, J. Molnar, J. Palinkas, Z. Szillasi 
University of Debrecen, Debrecen, Hungary

J. Karancsi, P. Raics, Z.L. Trocsanyi, B. Ujvari

Panjab University, Chandigarh, India

S.B. Beri, V. Bhatnagar, N. Dhingra, R. Gupta, M. Kaur, M.Z. Mehta, N. Nishu, L.K. Saini, A. Sharma, J. Singh

University of Delhi, Delhi, India

Ashok Kumar, Arun Kumar, S. Ahuja, A. Bhardwaj, B.C. Choudhary, S. Malhotra, M. Naimuddin, K. Ranjan, V. Sharma, R.K. Shivpuri

Saha Institute of Nuclear Physics, Kolkata, India

S. Banerjee, S. Bhattacharya, S. Dutta, B. Gomber, Sa. Jain, Sh. Jain, R. Khurana, S. Sarkar, M. Sharan

Bhabha Atomic Research Centre, Mumbai, India

A. Abdulsalam, R.K. Choudhury, D. Dutta, S. Kailas, V. Kumar, P. Mehta, A.K. Mohanty ${ }^{5}$, L.M. Pant, P. Shukla

Tata Institute of Fundamental Research - EHEP, Mumbai, India

T. Aziz, S. Ganguly, M. Guchait ${ }^{20}$, M. Maity ${ }^{21}$, G. Majumder, K. Mazumdar, G.B. Mohanty, B. Parida, K. Sudhakar, N. Wickramage

Tata Institute of Fundamental Research - HECR, Mumbai, India

S. Banerjee, S. Dugad

Institute for Research in Fundamental Sciences (IPM), Tehran, Iran

H. Arfaei, H. Bakhshiansohi ${ }^{22}$, S.M. Etesami ${ }^{23}$, A. Fahim ${ }^{22}$, M. Hashemi, H. Hesari, A. Jafari ${ }^{22}$, M. Khakzad, M. Mohammadi Najafabadi, S. Paktinat Mehdiabadi, B. Safarzadeh ${ }^{24}$, M. Zeinali ${ }^{23}$

INFN Sezione di Bari ${ }^{a}$, Università di Bari ${ }^{b}$, Politecnico di Bari ${ }^{c}$, Bari, Italy M. Abbrescia $^{a, b}$, L. Barbone ${ }^{a, b}$, C. Calabria ${ }^{a, b, 5}$, S.S. Chhibra ${ }^{a, b}$, A. Colaleo ${ }^{a}$, D. Creanza ${ }^{a, c}$, N. De Filippis ${ }^{a, c, 5}$, M. De Palma ${ }^{a, b}$, L. Fiore $^{a}$, G. Iaselli ${ }^{a, c}$, L. Lusito ${ }^{a, b}$, G. Maggi ${ }^{a, c}$, M. Maggi ${ }^{a}$, B. Marangelli ${ }^{a, b}$, S. My ${ }^{a, c}$, S. $\mathrm{Nuzzo}^{a, b}$, N. Pacifico ${ }^{a, b}$,

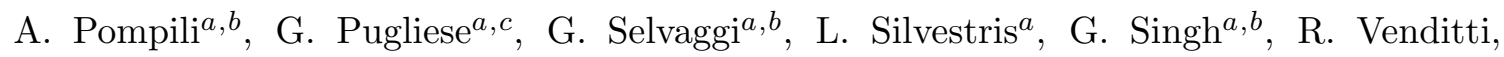
G. Zito ${ }^{a}$

INFN Sezione di Bologna ${ }^{a}$, Università di Bologna ${ }^{b}$, Bologna, Italy

G. Abbiendi ${ }^{a}$, A.C. Benvenuti ${ }^{a}$, D. Bonacorsi ${ }^{a, b}$, S. Braibant-Giacomelli ${ }^{a, b}$, L. Brigliadori ${ }^{a, b}$, P. Capiluppi ${ }^{a, b}$, A. Castro ${ }^{a, b}$, F.R. Cavallo ${ }^{a}$, M. Cuffiani ${ }^{a, b}$, G.M. Dallavalle ${ }^{a}$, F. Fabbri ${ }^{a}$, A. Fanfani ${ }^{a, b}$, D. Fasanella ${ }^{a, b, 5}$, P. Giacomelli ${ }^{a}$, C. Grandi ${ }^{a}$, L. Guiducci ${ }^{a}, b$, S. Marcellini ${ }^{a}$, G. Masetti ${ }^{a}$, M. Meneghelli ${ }^{a, b, 5}$, A. Montanari ${ }^{a}$,

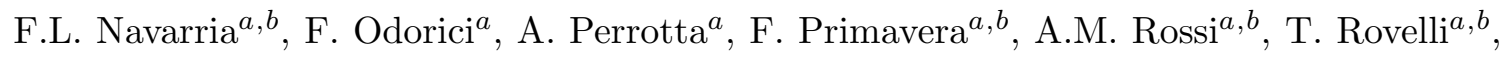
G. Siroli ${ }^{a, b}$, R. Travaglini ${ }^{a, b}$

INFN Sezione di Catania ${ }^{a}$, Università di Catania ${ }^{b}$, Catania, Italy

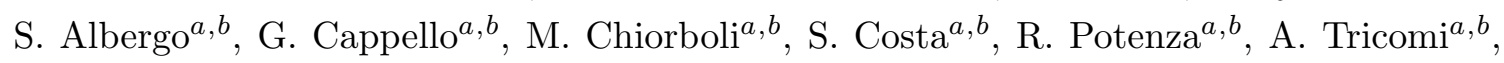
C. Tuve $\mathrm{e}^{a, b}$ 
INFN Sezione di Firenze ${ }^{a}$, Università di Firenze ${ }^{b}$, Firenze, Italy

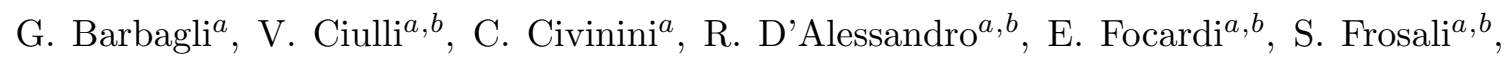

E. Gallo ${ }^{a}$, S. Gonzi ${ }^{a}, b$, M. Meschini ${ }^{a}$, S. Paoletti ${ }^{a}$, G. Sguazzoni $^{a}$, A. Tropiano ${ }^{a}$

INFN Laboratori Nazionali di Frascati, Frascati, Italy

L. Benussi, S. Bianco, S. Colafranceschi ${ }^{25}$, F. Fabbri, D. Piccolo

INFN Sezione di Genova, Genova, Italy

P. Fabbricatore, R. Musenich, S. Tosi

INFN Sezione di Milano-Bicocca ${ }^{a}$, Università di Milano-Bicocca ${ }^{b}$, Milano, Italy

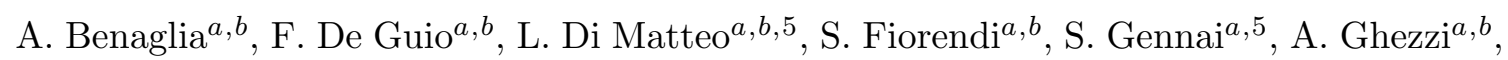
S. Malvezzi ${ }^{a}$, R.A. Manzoni ${ }^{a, b}$, A. Martelli ${ }^{a, b}$, A. Massironi ${ }^{a, b, 5}$, D. Menasce ${ }^{a}$, L. Moroni ${ }^{a}$, M. Paganoni ${ }^{a, b}$, D. Pedrini ${ }^{a}$, S. Ragazzi ${ }^{a, b}$, N. Redaelli ${ }^{a}$, S. Sala ${ }^{a}$, T. Tabarelli de Fatis ${ }^{a, b}$

INFN Sezione di Napoli ${ }^{a}$, Università di Napoli "Federico II" ${ }^{b}$, Napoli, Italy S. Buontempo ${ }^{a}$, C.A. Carrillo Montoya ${ }^{a}$, N. Cavallo ${ }^{a, 26}$, A. De Cosa ${ }^{a, b, 5}$, O. Dogangun ${ }^{a, b}$,

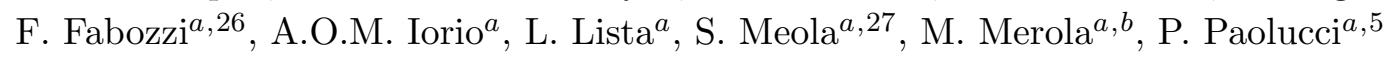

INFN Sezione di Padova ${ }^{a}$, Università di Padova ${ }^{b}$, Università di Trento (Trento) ${ }^{c}$, Padova, Italy

P. Azzi ${ }^{a}$, N. Bacchetta ${ }^{a, 5}$, P. Bellan ${ }^{a, b}$, D. Bisello ${ }^{a, b}$, A. Branca ${ }^{a, 5}$, P. Checchia ${ }^{a}$, T. Dorigo ${ }^{a}$, U. Dosselli ${ }^{a}$, F. Gasparini ${ }^{a}, b$, A. Gozzelino ${ }^{a}$, K. Kanishchev ${ }^{a, c}$, S. Lacaprara ${ }^{a}$, $^{a}$

I. Lazzizzera ${ }^{a, c}$, M. Margoni ${ }^{a, b}$, A.T. Meneguzzo ${ }^{a, b}$, M. Nespolo ${ }^{a, 5}$, J. Pazzini ${ }^{a}$, N. Pozzobon ${ }^{a, b}$, P. Ronchese ${ }^{a, b}$, F. Simonetto ${ }^{a, b}$, E. Torassa ${ }^{a}$, M. Tosi ${ }^{a, b, 5}$, S. Vanini ${ }^{a, b}$, P. Zotto ${ }^{a, b}$, G. Zumerle ${ }^{a, b}$

INFN Sezione di Pavia ${ }^{a}$, Università di Pavia ${ }^{b}$, Pavia, Italy

M. Gabusi ${ }^{a}, b$, S.P. Ratti ${ }^{a, b}$, C. Riccardi ${ }^{a, b}$, P. Torre ${ }^{a, b}$, P. Vitulo ${ }^{a, b}$

INFN Sezione di Perugia ${ }^{a}$, Università di Perugia ${ }^{b}$, Perugia, Italy

M. Biasini ${ }^{a}, b$, G.M. Bilei ${ }^{a}$, L. Fanò ${ }^{a}, b$, P. Lariccia ${ }^{a, b}$, A. Lucaroni ${ }^{a, b, 5}$, G. Mantovani $^{a, b}$, M. Menichelli ${ }^{a}$, A. Nappi ${ }^{a, b \dagger}$, F. Romeo ${ }^{a, b}$, A. Saha ${ }^{a}$, A. Santocchia ${ }^{a, b}$, A. Spiezia ${ }^{a, b}$, S. Taroni ${ }^{a, b}$

INFN Sezione di Pisa ${ }^{a}$, Università di Pisa ${ }^{b}$, Scuola Normale Superiore di Pisa ${ }^{c}$, Pisa, Italy

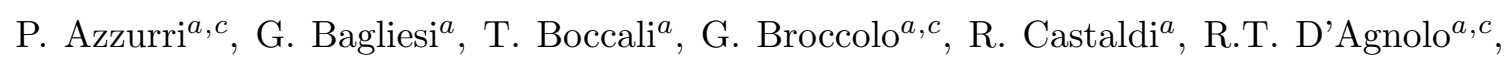

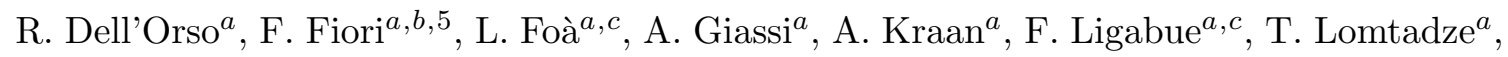
L. Martini ${ }^{a, 28}$, A. Messineo ${ }^{a, b}$, F. Palla ${ }^{a}$, A. Rizzi ${ }^{a, b}$, A.T. Serban ${ }^{a, 29}$, P. Spagnolo ${ }^{a}$,

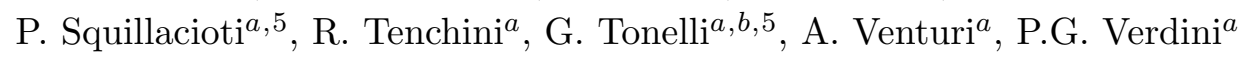

INFN Sezione di Roma ${ }^{a}$, Università di Roma "La Sapienza" ${ }^{b}$, Roma, Italy

L. Barone ${ }^{a, b}$, F. Cavallari ${ }^{a}$, D. Del Re $e^{a, b}$, M. Diemoz ${ }^{a}$, C. Fanelli, M. Grassi ${ }^{a, b, 5}$, E. Longo ${ }^{a, b}$, P. Meridiani ${ }^{a, 5}$, F. Micheli ${ }^{a, b}$, S. Nourbakhsh ${ }^{a, b}$, G. Organtini ${ }^{a, b}$, R. Paramatti ${ }^{a}$, S. Rahatlou $^{a, b}$, M. Sigamani ${ }^{a}$, L. Soffi $^{a, b}$ 
INFN Sezione di Torino ${ }^{a}$, Università di Torino ${ }^{b}$, Università del Piemonte Orientale (Novara) ${ }^{c}$, Torino, Italy

N. Amapane ${ }^{a, b}$, R. Arcidiacono ${ }^{a, c}, \mathrm{~S}$. Argiro $^{a, b}$, M. Arneodo $^{a, c}$, C. Bino $^{a}$, N. Cartiglia ${ }^{a}$, M. Costa ${ }^{a, b}$, P. De Remigis ${ }^{a}$, N. Demaria ${ }^{a}$, C. Mariotti ${ }^{a}, 5$, S. Maselli ${ }^{a}$, E. Migliore ${ }^{a, b}$, V. Monaco ${ }^{a, b}$, M. Musich ${ }^{a, 5}$, M.M. Obertino ${ }^{a, c}$, N. Pastrone ${ }^{a}$, M. Pelliccioni ${ }^{a}$, A. Potenza $^{a, b}$, A. Romero $^{a, b}$, M. Ruspa $^{a, c}$, R. Sacchi $^{a}, b$, A. Solano $^{a, b}$, A. Staiano $^{a}$, A. Vilela Pereira $^{a}$

INFN Sezione di Trieste ${ }^{a}$, Università di Trieste ${ }^{b}$, Trieste, Italy

S. Belforte ${ }^{a}$, V. Candelise ${ }^{a, b}$, F. Cossutti ${ }^{a}$, G. Della Ricca ${ }^{a, b}$, B. Gobbo $^{a}$, M. Marone ${ }^{a, b, 5}$, D. Montanino ${ }^{a, b, 5}$, A. Penzo $^{a}$, A. Schizzi ${ }^{a, b}$

Kangwon National University, Chunchon, Korea

S.G. Heo, T.Y. Kim, S.K. Nam

Kyungpook National University, Daegu, Korea

S. Chang, D.H. Kim, G.N. Kim, D.J. Kong, H. Park, S.R. Ro, D.C. Son, T. Son

Chonnam National University, Institute for Universe and Elementary Particles, Kwangju, Korea

J.Y. Kim, Zero J. Kim, S. Song

Korea University, Seoul, Korea

S. Choi, D. Gyun, B. Hong, M. Jo, H. Kim, T.J. Kim, K.S. Lee, D.H. Moon, S.K. Park

University of Seoul, Seoul, Korea

M. Choi, J.H. Kim, C. Park, I.C. Park, S. Park, G. Ryu

Sungkyunkwan University, Suwon, Korea

Y. Cho, Y. Choi, Y.K. Choi, J. Goh, M.S. Kim, E. Kwon, B. Lee, J. Lee, S. Lee, H. Seo, I. $\mathrm{Yu}$

Vilnius University, Vilnius, Lithuania

M.J. Bilinskas, I. Grigelionis, M. Janulis, A. Juodagalvis

Centro de Investigacion y de Estudios Avanzados del IPN, Mexico City, Mexico

H. Castilla-Valdez, E. De La Cruz-Burelo, I. Heredia-de La Cruz, R. Lopez-Fernandez,

R. Magaña Villalba, J. Martínez-Ortega, A. Sánchez-Hernández, L.M. Villasenor-Cendejas

Universidad Iberoamericana, Mexico City, Mexico

S. Carrillo Moreno, F. Vazquez Valencia

Benemerita Universidad Autonoma de Puebla, Puebla, Mexico

H.A. Salazar Ibarguen

Universidad Autónoma de San Luis Potosí, San Luis Potosí, Mexico

E. Casimiro Linares, A. Morelos Pineda, M.A. Reyes-Santos

University of Auckland, Auckland, New Zealand

D. Krofcheck 
University of Canterbury, Christchurch, New Zealand

A.J. Bell, P.H. Butler, R. Doesburg, S. Reucroft, H. Silverwood

National Centre for Physics, Quaid-I-Azam University, Islamabad, Pakistan

M. Ahmad, M.H. Ansari, M.I. Asghar, H.R. Hoorani, S. Khalid, W.A. Khan, T. Khurshid, S. Qazi, M.A. Shah, M. Shoaib

Institute of Experimental Physics, Faculty of Physics, University of Warsaw, Warsaw, Poland

G. Brona, K. Bunkowski, M. Cwiok, W. Dominik, K. Doroba, A. Kalinowski, M. Konecki, J. Krolikowski

Soltan Institute for Nuclear Studies, Warsaw, Poland

H. Bialkowska, B. Boimska, T. Frueboes, R. Gokieli, M. Górski, M. Kazana, K. Nawrocki,

K. Romanowska-Rybinska, M. Szleper, G. Wrochna, P. Zalewski

Laboratório de Instrumentação e Física Experimental de Partículas, Lisboa, Portugal

N. Almeida, P. Bargassa, A. David, P. Faccioli, P.G. Ferreira Parracho, M. Gallinaro, J. Seixas, J. Varela, P. Vischia

Joint Institute for Nuclear Research, Dubna, Russia

I. Belotelov, P. Bunin, I. Golutvin, A. Kamenev, V. Karjavin, V. Konoplyanikov, G. Kozlov, A. Lanev, A. Malakhov, P. Moisenz, V. Palichik, V. Perelygin, M. Savina, S. Shmatov, V. Smirnov, A. Volodko, A. Zarubin

Petersburg Nuclear Physics Institute, Gatchina (St Petersburg), Russia

S. Evstyukhin, V. Golovtsov, Y. Ivanov, V. Kim, P. Levchenko, V. Murzin, V. Oreshkin, I. Smirnov, V. Sulimov, L. Uvarov, S. Vavilov, A. Vorobyev, An. Vorobyev

Institute for Nuclear Research, Moscow, Russia

Yu. Andreev, A. Dermenev, S. Gninenko, N. Golubev, M. Kirsanov, N. Krasnikov, V. Matveev, A. Pashenkov, D. Tlisov, A. Toropin

Institute for Theoretical and Experimental Physics, Moscow, Russia

V. Epshteyn, M. Erofeeva, V. Gavrilov, M. Kossov, N. Lychkovskaya, V. Popov, G. Safronov, S. Semenov, V. Stolin, E. Vlasov, A. Zhokin

Moscow State University, Moscow, Russia

A. Belyaev, E. Boos, M. Dubinin ${ }^{4}$, L. Dudko, A. Ershov, A. Gribushin, V. Klyukhin, O. Kodolova, I. Lokhtin, A. Markina, S. Obraztsov, M. Perfilov, S. Petrushanko, A. Popov, L. Sarycheva ${ }^{\dagger}$, V. Savrin, A. Snigirev

P.N. Lebedev Physical Institute, Moscow, Russia

V. Andreev, M. Azarkin, I. Dremin, M. Kirakosyan, A. Leonidov, G. Mesyats, S.V. Rusakov, A. Vinogradov 
State Research Center of Russian Federation, Institute for High Energy Physics, Protvino, Russia

I. Azhgirey, I. Bayshev, S. Bitioukov, V. Grishin ${ }^{5}$, V. Kachanov, D. Konstantinov, A. Korablev, V. Krychkine, V. Petrov, R. Ryutin, A. Sobol, L. Tourtchanovitch, S. Troshin, N. Tyurin, A. Uzunian, A. Volkov

University of Belgrade, Faculty of Physics and Vinca Institute of Nuclear Sciences, Belgrade, Serbia

P. Adzic ${ }^{30}$, M. Djordjevic, M. Ekmedzic, D. Krpic ${ }^{30}$, J. Milosevic

Centro de Investigaciones Energéticas Medioambientales y Tecnológicas (CIEMAT), Madrid, Spain

M. Aguilar-Benitez, J. Alcaraz Maestre, P. Arce, C. Battilana, E. Calvo, M. Cerrada, M. Chamizo Llatas, N. Colino, B. De La Cruz, A. Delgado Peris, D. Domínguez Vázquez, C. Fernandez Bedoya, J.P. Fernández Ramos, A. Ferrando, J. Flix, M.C. Fouz, P. GarciaAbia, O. Gonzalez Lopez, S. Goy Lopez, J.M. Hernandez, M.I. Josa, G. Merino, J. Puerta Pelayo, A. Quintario Olmeda, I. Redondo, L. Romero, J. Santaolalla, M.S. Soares, C. Willmott

Universidad Autónoma de Madrid, Madrid, Spain

C. Albajar, G. Codispoti, J.F. de Trocóniz

Universidad de Oviedo, Oviedo, Spain

H. Brun, J. Cuevas, J. Fernandez Menendez, S. Folgueras, I. Gonzalez Caballero, L. Lloret Iglesias, J. Piedra Gomez ${ }^{31}$

Instituto de Física de Cantabria (IFCA), CSIC-Universidad de Cantabria, Santander, Spain

J.A. Brochero Cifuentes, I.J. Cabrillo, A. Calderon, S.H. Chuang, J. Duarte Campderros, M. Felcini ${ }^{32}$, M. Fernandez, G. Gomez, J. Gonzalez Sanchez, A. Graziano, C. Jorda, A. Lopez Virto, J. Marco, R. Marco, C. Martinez Rivero, F. Matorras, F.J. Munoz Sanchez,

T. Rodrigo, A.Y. Rodríguez-Marrero, A. Ruiz-Jimeno, L. Scodellaro, M. Sobron Sanudo, I. Vila, R. Vilar Cortabitarte

CERN, European Organization for Nuclear Research, Geneva, Switzerland

D. Abbaneo, E. Auffray, G. Auzinger, M. Bachtis, P. Baillon, A.H. Ball, D. Barney, J.F. Benitez, C. Bernet ${ }^{6}$, G. Bianchi, P. Bloch, A. Bocci, A. Bonato, C. Botta, H. Breuker, T. Camporesi, G. Cerminara, T. Christiansen, J.A. Coarasa Perez, D. D'Enterria, A. Dabrowski, A. De Roeck, S. Di Guida, M. Dobson, N. Dupont-Sagorin, A. ElliottPeisert, B. Frisch, W. Funk, G. Georgiou, M. Giffels, D. Gigi, K. Gill, D. Giordano, M. Giunta, F. Glege, R. Gomez-Reino Garrido, P. Govoni, S. Gowdy, R. Guida, M. Hansen, P. Harris, C. Hartl, J. Harvey, B. Hegner, A. Hinzmann, V. Innocente, P. Janot, K. Kaadze, E. Karavakis, K. Kousouris, P. Lecoq, Y.-J. Lee, P. Lenzi, C. Lourenço, N. Magini, T. Mäki, M. Malberti, L. Malgeri, M. Mannelli, L. Masetti, F. Meijers, S. Mersi, E. Meschi, R. Moser, M.U. Mozer, M. Mulders, P. Musella, E. Nesvold, T. Orimoto, L. Orsini, E. Palencia Cortezon, E. Perez, L. Perrozzi, A. Petrilli, A. Pfeiffer, M. Pierini, M. Pimiä, D. Piparo, 
G. Polese, L. Quertenmont, A. Racz, W. Reece, J. Rodrigues Antunes, G. Rolandi ${ }^{33}$, C. Rovelli ${ }^{34}$, M. Rovere, H. Sakulin, F. Santanastasio, C. Schäfer, C. Schwick, I. Segoni, S. Sekmen, A. Sharma, P. Siegrist, P. Silva, M. Simon, P. Sphicas ${ }^{35}$, D. Spiga, A. Tsirou, G.I. Veres ${ }^{19}$, J.R. Vlimant, H.K. Wöhri, S.D. Worm ${ }^{36}$, W.D. Zeuner

\section{Paul Scherrer Institut, Villigen, Switzerland}

W. Bertl, K. Deiters, W. Erdmann, K. Gabathuler, R. Horisberger, Q. Ingram, H.C. Kaestli, S. König, D. Kotlinski, U. Langenegger, F. Meier, D. Renker, T. Rohe, J. Sibille ${ }^{37}$

Institute for Particle Physics, ETH Zurich, Zurich, Switzerland

L. Bäni, P. Bortignon, M.A. Buchmann, B. Casal, N. Chanon, A. Deisher, G. Dissertori, M. Dittmar, M. Donegà, M. Dünser, J. Eugster, K. Freudenreich, C. Grab, D. Hits, P. Lecomte, W. Lustermann, A.C. Marini, P. Martinez Ruiz del Arbol, N. Mohr, F. Moortgat, C. Nägeli ${ }^{38}$, P. Nef, F. Nessi-Tedaldi, F. Pandolfi, L. Pape, F. Pauss, M. Peruzzi, F.J. Ronga, M. Rossini, L. Sala, A.K. Sanchez, A. Starodumov ${ }^{39}$, B. Stieger, M. Takahashi, L. Tauscher ${ }^{\dagger}$, A. Thea, K. Theofilatos, D. Treille, C. Urscheler, R. Wallny, H.A. Weber, L. Wehrli

\section{Universität Zürich, Zurich, Switzerland}

C. Amsler, V. Chiochia, S. De Visscher, C. Favaro, M. Ivova Rikova, B. Millan Mejias, P. Otiougova, P. Robmann, H. Snoek, S. Tupputi, M. Verzetti

\section{National Central University, Chung-Li, Taiwan}

Y.H. Chang, K.H. Chen, C.M. Kuo, S.W. Li, W. Lin, Z.K. Liu, Y.J. Lu, D. Mekterovic, A.P. Singh, R. Volpe, S.S. Yu

\section{National Taiwan University (NTU), Taipei, Taiwan}

P. Bartalini, P. Chang, Y.H. Chang, Y.W. Chang, Y. Chao, K.F. Chen, C. Dietz, U. Grundler, W.-S. Hou, Y. Hsiung, K.Y. Kao, Y.J. Lei, R.-S. Lu, D. Majumder, E. Petrakou, X. Shi, J.G. Shiu, Y.M. Tzeng, X. Wan, M. Wang

\section{Cukurova University, Adana, Turkey}

A. Adiguzel, M.N. Bakirci ${ }^{40}$, S. Cerci ${ }^{41}$, C. Dozen, I. Dumanoglu, E. Eskut, S. Girgis, G. Gokbulut, E. Gurpinar, I. Hos, E.E. Kangal, T. Karaman, G. Karapinar ${ }^{42}$, A. Kayis Topaksu, G. Onengut, K. Ozdemir, S. Ozturk ${ }^{43}$, A. Polatoz, K. Sogut ${ }^{44}$, D. Sunar Cerci ${ }^{41}$, B. Tali ${ }^{41}$, H. Topakli ${ }^{40}$, L.N. Vergili, M. Vergili

Middle East Technical University, Physics Department, Ankara, Turkey I.V. Akin, T. Aliev, B. Bilin, S. Bilmis, M. Deniz, H. Gamsizkan, A.M. Guler, K. Ocalan, A. Ozpineci, M. Serin, R. Sever, U.E. Surat, M. Yalvac, E. Yildirim, M. Zeyrek

\section{Bogazici University, Istanbul, Turkey}

E. Gülmez, B. Isildak ${ }^{45}$, M. Kaya ${ }^{46}$, O. Kaya ${ }^{46}$, S. Ozkorucuklu ${ }^{47}$, N. Sonmez ${ }^{48}$

\section{Istanbul Technical University, Istanbul, Turkey}

K. Cankocak 
National Scientific Center, Kharkov Institute of Physics and Technology, Kharkov, Ukraine

L. Levchuk

\section{University of Bristol, Bristol, United Kingdom}

F. Bostock, J.J. Brooke, E. Clement, D. Cussans, H. Flacher, R. Frazier, J. Goldstein, M. Grimes, G.P. Heath, H.F. Heath, L. Kreczko, S. Metson, D.M. Newbold ${ }^{36}$, K. Nirunpong, A. Poll, S. Senkin, V.J. Smith, T. Williams

\section{Rutherford Appleton Laboratory, Didcot, United Kingdom}

L. Basso ${ }^{49}$, K.W. Bell, A. Belyaev ${ }^{49}$, C. Brew, R.M. Brown, D.J.A. Cockerill, J.A. Coughlan, K. Harder, S. Harper, J. Jackson, B.W. Kennedy, E. Olaiya, D. Petyt, B.C. RadburnSmith, C.H. Shepherd-Themistocleous, I.R. Tomalin, W.J. Womersley

\section{Imperial College, London, United Kingdom}

R. Bainbridge, G. Ball, R. Beuselinck, O. Buchmuller, D. Colling, N. Cripps, M. Cutajar, P. Dauncey, G. Davies, M. Della Negra, W. Ferguson, J. Fulcher, D. Futyan, A. Gilbert, A. Guneratne Bryer, G. Hall, Z. Hatherell, J. Hays, G. Iles, M. Jarvis, G. Karapostoli, L. Lyons, A.-M. Magnan, J. Marrouche, B. Mathias, R. Nandi, J. Nash, A. Nikitenko ${ }^{39}$, A. Papageorgiou, J. Pela, M. Pesaresi, K. Petridis, M. Pioppi ${ }^{50}$, D.M. Raymond, S. Rogerson, A. Rose, M.J. Ryan, C. Seez, P. Sharp ${ }^{\dagger}$, A. Sparrow, M. Stoye, A. Tapper, M. Vazquez Acosta, T. Virdee, S. Wakefield, N. Wardle, T. Whyntie

\section{Brunel University, Uxbridge, United Kingdom}

M. Chadwick, J.E. Cole, P.R. Hobson, A. Khan, P. Kyberd, D. Leggat, D. Leslie, W. Martin, I.D. Reid, P. Symonds, L. Teodorescu, M. Turner

Baylor University, Waco, USA

K. Hatakeyama, H. Liu, T. Scarborough

The University of Alabama, Tuscaloosa, USA

O. Charaf, C. Henderson, P. Rumerio

\section{Boston University, Boston, USA}

A. Avetisyan, T. Bose, C. Fantasia, A. Heister, J. St. John, P. Lawson, D. Lazic, J. Rohlf, D. Sperka, L. Sulak

\section{Brown University, Providence, USA}

J. Alimena, S. Bhattacharya, D. Cutts, A. Ferapontov, U. Heintz, S. Jabeen, G. Kukartsev, E. Laird, G. Landsberg, M. Luk, M. Narain, D. Nguyen, M. Segala, T. Sinthuprasith, T. Speer, K.V. Tsang

\section{University of California, Davis, Davis, USA}

R. Breedon, G. Breto, M. Calderon De La Barca Sanchez, S. Chauhan, M. Chertok, J. Conway, R. Conway, P.T. Cox, J. Dolen, R. Erbacher, M. Gardner, R. Houtz, W. Ko, A. Kopecky, R. Lander, T. Miceli, D. Pellett, F. Ricci-tam, B. Rutherford, M. Searle, J. Smith, M. Squires, M. Tripathi, R. Vasquez Sierra 
University of California, Los Angeles, Los Angeles, USA

V. Andreev, D. Cline, R. Cousins, J. Duris, S. Erhan, P. Everaerts, C. Farrell, J. Hauser, M. Ignatenko, C. Jarvis, C. Plager, G. Rakness, P. Schlein ${ }^{\dagger}$, P. Traczyk, V. Valuev, M. Weber

\section{University of California, Riverside, Riverside, USA}

J. Babb, R. Clare, M.E. Dinardo, J. Ellison, J.W. Gary, F. Giordano, G. Hanson, G.Y. Jeng ${ }^{51}$, H. Liu, O.R. Long, A. Luthra, H. Nguyen, S. Paramesvaran, J. Sturdy, S. Sumowidagdo, R. Wilken, S. Wimpenny

\section{University of California, San Diego, La Jolla, USA}

W. Andrews, J.G. Branson, G.B. Cerati, S. Cittolin, D. Evans, F. Golf, A. Holzner, R. Kelley, M. Lebourgeois, J. Letts, I. Macneill, B. Mangano, S. Padhi, C. Palmer, G. Petrucciani, M. Pieri, M. Sani, V. Sharma, S. Simon, E. Sudano, M. Tadel, Y. Tu, A. Vartak, S. Wasserbaech ${ }^{52}$, F. Würthwein, A. Yagil, J. Yoo

\section{University of California, Santa Barbara, Santa Barbara, USA}

D. Barge, R. Bellan, C. Campagnari, M. D’Alfonso, T. Danielson, K. Flowers, P. Geffert, J. Incandela, C. Justus, P. Kalavase, S.A. Koay, D. Kovalskyi, V. Krutelyov, S. Lowette, N. Mccoll, V. Pavlunin, F. Rebassoo, J. Ribnik, J. Richman, R. Rossin, D. Stuart, W. To, C. West

\section{California Institute of Technology, Pasadena, USA}

A. Apresyan, A. Bornheim, Y. Chen, E. Di Marco, J. Duarte, M. Gataullin, Y. Ma, A. Mott, H.B. Newman, C. Rogan, M. Spiropulu, V. Timciuc, J. Veverka, R. Wilkinson, S. Xie, Y. Yang, R.Y. Zhu

\section{Carnegie Mellon University, Pittsburgh, USA}

B. Akgun, V. Azzolini, A. Calamba, R. Carroll, T. Ferguson, Y. Iiyama, D.W. Jang, Y.F. Liu, M. Paulini, H. Vogel, I. Vorobiev

\section{University of Colorado at Boulder, Boulder, USA}

J.P. Cumalat, B.R. Drell, C.J. Edelmaier, W.T. Ford, A. Gaz, B. Heyburn, E. Luiggi Lopez, J.G. Smith, K. Stenson, K.A. Ulmer, S.R. Wagner

\section{Cornell University, Ithaca, USA}

J. Alexander, A. Chatterjee, N. Eggert, L.K. Gibbons, B. Heltsley, A. Khukhunaishvili, B. Kreis, N. Mirman, G. Nicolas Kaufman, J.R. Patterson, A. Ryd, E. Salvati, W. Sun, W.D. Teo, J. Thom, J. Thompson, J. Tucker, J. Vaughan, Y. Weng, L. Winstrom, P. Wittich

\section{Fairfield University, Fairfield, USA}

D. Winn

\section{Fermi National Accelerator Laboratory, Batavia, USA}

S. Abdullin, M. Albrow, J. Anderson, L.A.T. Bauerdick, A. Beretvas, J. Berryhill, P.C. Bhat, I. Bloch, K. Burkett, J.N. Butler, V. Chetluru, H.W.K. Cheung, F. Chlebana, 
V.D. Elvira, I. Fisk, J. Freeman, Y. Gao, D. Green, O. Gutsche, J. Hanlon, R.M. Harris, J. Hirschauer, B. Hooberman, S. Jindariani, M. Johnson, U. Joshi, B. Kilminster, B. Klima, S. Kunori, S. Kwan, C. Leonidopoulos, J. Linacre, D. Lincoln, R. Lipton, J. Lykken, K. Maeshima, J.M. Marraffino, S. Maruyama, D. Mason, P. McBride, K. Mishra, S. Mrenna, Y. Musienko ${ }^{53}$, C. Newman-Holmes, V. O'Dell, O. Prokofyev, E. SextonKennedy, S. Sharma, W.J. Spalding, L. Spiegel, P. Tan, L. Taylor, S. Tkaczyk, N.V. Tran, L. Uplegger, E.W. Vaandering, R. Vidal, J. Whitmore, W. Wu, F. Yang, F. Yumiceva, J.C. Yun

\section{University of Florida, Gainesville, USA}

D. Acosta, P. Avery, D. Bourilkov, M. Chen, T. Cheng, S. Das, M. De Gruttola, G.P. Di Giovanni, D. Dobur, A. Drozdetskiy, R.D. Field, M. Fisher, Y. Fu, I.K. Furic, J. Gartner, J. Hugon, B. Kim, J. Konigsberg, A. Korytov, A. Kropivnitskaya, T. Kypreos, J.F. Low, K. Matchev, P. Milenovic ${ }^{54}$, G. Mitselmakher, L. Muniz, R. Remington, A. Rinkevicius, P. Sellers, N. Skhirtladze, M. Snowball, J. Yelton, M. Zakaria

Florida International University, Miami, USA

V. Gaultney, S. Hewamanage, L.M. Lebolo, S. Linn, P. Markowitz, G. Martinez, J.L. Rodriguez

Florida State University, Tallahassee, USA

T. Adams, A. Askew, J. Bochenek, J. Chen, B. Diamond, S.V. Gleyzer, J. Haas, S. Hagopian, V. Hagopian, M. Jenkins, K.F. Johnson, H. Prosper, V. Veeraraghavan, M. Weinberg

\section{Florida Institute of Technology, Melbourne, USA}

M.M. Baarmand, B. Dorney, M. Hohlmann, H. Kalakhety, I. Vodopiyanov

University of Illinois at Chicago (UIC), Chicago, USA

M.R. Adams, I.M. Anghel, L. Apanasevich, Y. Bai, V.E. Bazterra, R.R. Betts, I. Bucinskaite, J. Callner, R. Cavanaugh, O. Evdokimov, L. Gauthier, C.E. Gerber, D.J. Hofman, S. Khalatyan, F. Lacroix, M. Malek, C. O'Brien, C. Silkworth, D. Strom, N. Varelas

\section{The University of Iowa, Iowa City, USA}

U. Akgun, E.A. Albayrak, B. Bilki ${ }^{55}$, W. Clarida, F. Duru, S. Griffiths, J.-P. Merlo, H. Mermerkaya ${ }^{56}$, A. Mestvirishvili, A. Moeller, J. Nachtman, C.R. Newsom, E. Norbeck, Y. Onel, F. Ozok, S. Sen, E. Tiras, J. Wetzel, T. Yetkin, K. Yi

\section{Johns Hopkins University, Baltimore, USA}

B.A. Barnett, B. Blumenfeld, S. Bolognesi, D. Fehling, G. Giurgiu, A.V. Gritsan, Z.J. Guo, G. Hu, P. Maksimovic, S. Rappoccio, M. Swartz, A. Whitbeck

\section{The University of Kansas, Lawrence, USA}

P. Baringer, A. Bean, G. Benelli, O. Grachov, R.P. Kenny Iii, M. Murray, D. Noonan, S. Sanders, R. Stringer, G. Tinti, J.S. Wood, V. Zhukova 
Kansas State University, Manhattan, USA

A.F. Barfuss, T. Bolton, I. Chakaberia, A. Ivanov, S. Khalil, M. Makouski, Y. Maravin, S. Shrestha, I. Svintradze

Lawrence Livermore National Laboratory, Livermore, USA

J. Gronberg, D. Lange, D. Wright

University of Maryland, College Park, USA

A. Baden, M. Boutemeur, B. Calvert, S.C. Eno, J.A. Gomez, N.J. Hadley, R.G. Kellogg, M. Kirn, T. Kolberg, Y. Lu, M. Marionneau, A.C. Mignerey, K. Pedro, A. Peterman, A. Skuja, J. Temple, M.B. Tonjes, S.C. Tonwar, E. Twedt

\section{Massachusetts Institute of Technology, Cambridge, USA}

A. Apyan, G. Bauer, J. Bendavid, W. Busza, E. Butz, I.A. Cali, M. Chan, V. Dutta, G. Gomez Ceballos, M. Goncharov, K.A. Hahn, Y. Kim, M. Klute, K. Krajczar ${ }^{57}$, W. Li, P.D. Luckey, T. Ma, S. Nahn, C. Paus, D. Ralph, C. Roland, G. Roland, M. Rudolph, G.S.F. Stephans, F. Stöckli, K. Sumorok, K. Sung, D. Velicanu, E.A. Wenger, R. Wolf, B. Wyslouch, M. Yang, Y. Yilmaz, A.S. Yoon, M. Zanetti

\section{University of Minnesota, Minneapolis, USA}

S.I. Cooper, B. Dahmes, A. De Benedetti, G. Franzoni, A. Gude, S.C. Kao, K. Klapoetke, Y. Kubota, J. Mans, N. Pastika, R. Rusack, M. Sasseville, A. Singovsky, N. Tambe, J. Turkewitz

University of Mississippi, University, USA

L.M. Cremaldi, R. Kroeger, L. Perera, R. Rahmat, D.A. Sanders

University of Nebraska-Lincoln, Lincoln, USA

E. Avdeeva, K. Bloom, S. Bose, J. Butt, D.R. Claes, A. Dominguez, M. Eads, J. Keller, I. Kravchenko, J. Lazo-Flores, H. Malbouisson, S. Malik, G.R. Snow

State University of New York at Buffalo, Buffalo, USA

U. Baur, A. Godshalk, I. Iashvili, S. Jain, A. Kharchilava, A. Kumar, S.P. Shipkowski, K. Smith

\section{Northeastern University, Boston, USA}

G. Alverson, E. Barberis, D. Baumgartel, M. Chasco, J. Haley, D. Nash, D. Trocino, D. Wood, J. Zhang

\section{Northwestern University, Evanston, USA}

A. Anastassov, A. Kubik, N. Mucia, N. Odell, R.A. Ofierzynski, B. Pollack, A. Pozdnyakov, M. Schmitt, S. Stoynev, M. Velasco, S. Won

\section{University of Notre Dame, Notre Dame, USA}

L. Antonelli, D. Berry, A. Brinkerhoff, M. Hildreth, C. Jessop, D.J. Karmgard, J. Kolb, K. Lannon, W. Luo, S. Lynch, N. Marinelli, D.M. Morse, T. Pearson, M. Planer, R. Ruchti, J. Slaunwhite, N. Valls, M. Wayne, M. Wolf 
The Ohio State University, Columbus, USA

B. Bylsma, L.S. Durkin, C. Hill, K. Kotov, T.Y. Ling, D. Puigh, M. Rodenburg, C. Vuosalo, G. Williams, B.L. Winer

\section{Princeton University, Princeton, USA}

N. Adam, E. Berry, P. Elmer, D. Gerbaudo, V. Halyo, P. Hebda, J. Hegeman, A. Hunt, P. Jindal, D. Lopes Pegna, P. Lujan, D. Marlow, T. Medvedeva, M. Mooney, J. Olsen, P. Piroué, X. Quan, A. Raval, B. Safdi, H. Saka, D. Stickland, C. Tully, J.S. Werner, A. Zuranski

\section{University of Puerto Rico, Mayaguez, USA}

J.G. Acosta, E. Brownson, X.T. Huang, A. Lopez, H. Mendez, S. Oliveros, J.E. Ramirez Vargas, A. Zatserklyaniy

Purdue University, West Lafayette, USA

E. Alagoz, V.E. Barnes, D. Benedetti, G. Bolla, D. Bortoletto, M. De Mattia, A. Everett, Z. Hu, M. Jones, O. Koybasi, M. Kress, A.T. Laasanen, N. Leonardo, V. Maroussov, P. Merkel, D.H. Miller, N. Neumeister, I. Shipsey, D. Silvers, A. Svyatkovskiy, M. Vidal Marono, H.D. Yoo, J. Zablocki, Y. Zheng

Purdue University Calumet, Hammond, USA

S. Guragain, N. Parashar

\section{Rice University, Houston, USA}

A. Adair, C. Boulahouache, K.M. Ecklund, F.J.M. Geurts, B.P. Padley, R. Redjimi, J. Roberts, J. Zabel

University of Rochester, Rochester, USA

B. Betchart, A. Bodek, Y.S. Chung, R. Covarelli, P. de Barbaro, R. Demina, Y. Eshaq, T. Ferbel, A. Garcia-Bellido, P. Goldenzweig, J. Han, A. Harel, D.C. Miner, D. Vishnevskiy, M. Zielinski

\section{The Rockefeller University, New York, USA}

A. Bhatti, R. Ciesielski, L. Demortier, K. Goulianos, G. Lungu, S. Malik, C. Mesropian

Rutgers, the State University of New Jersey, Piscataway, USA

S. Arora, A. Barker, J.P. Chou, C. Contreras-Campana, E. Contreras-Campana, D. Duggan, D. Ferencek, Y. Gershtein, R. Gray, E. Halkiadakis, D. Hidas, A. Lath, S. Panwalkar, M. Park, R. Patel, V. Rekovic, J. Robles, K. Rose, S. Salur, S. Schnetzer, C. Seitz, S. Somalwar, R. Stone, S. Thomas

University of Tennessee, Knoxville, USA

G. Cerizza, M. Hollingsworth, S. Spanier, Z.C. Yang, A. York

Texas A\&M University, College Station, USA

R. Eusebi, W. Flanagan, J. Gilmore, T. Kamon ${ }^{58}$, V. Khotilovich, R. Montalvo, I. Osipenkov, Y. Pakhotin, A. Perloff, J. Roe, A. Safonov, T. Sakuma, S. Sengupta, I. Suarez, A. Tatarinov, D. Toback 


\section{Texas Tech University, Lubbock, USA}

N. Akchurin, J. Damgov, C. Dragoiu, P.R. Dudero, C. Jeong, K. Kovitanggoon, S.W. Lee, T. Libeiro, Y. Roh, I. Volobouev

\section{Vanderbilt University, Nashville, USA}

E. Appelt, A.G. Delannoy, C. Florez, S. Greene, A. Gurrola, W. Johns, C. Johnston, P. Kurt, C. Maguire, A. Melo, M. Sharma, P. Sheldon, B. Snook, S. Tuo, J. Velkovska

\section{University of Virginia, Charlottesville, USA}

M.W. Arenton, M. Balazs, S. Boutle, B. Cox, B. Francis, J. Goodell, R. Hirosky, A. Ledovskoy, C. Lin, C. Neu, J. Wood, R. Yohay

\section{Wayne State University, Detroit, USA}

S. Gollapinni, R. Harr, P.E. Karchin, C. Kottachchi Kankanamge Don, P. Lamichhane, A. Sakharov

\section{University of Wisconsin, Madison, USA}

M. Anderson, D. Belknap, L. Borrello, D. Carlsmith, M. Cepeda, S. Dasu, L. Gray, K.S. Grogg, M. Grothe, R. Hall-Wilton, M. Herndon, A. Hervé, P. Klabbers, J. Klukas, A. Lanaro, C. Lazaridis, J. Leonard, R. Loveless, A. Mohapatra, I. Ojalvo, F. Palmonari, G.A. Pierro, I. Ross, A. Savin, W.H. Smith, J. Swanson

\section{$\dagger$ : Deceased}

1: Also at Vienna University of Technology, Vienna, Austria

2: Also at National Institute of Chemical Physics and Biophysics, Tallinn, Estonia

3: Also at Universidade Federal do ABC, Santo Andre, Brazil

4: Also at California Institute of Technology, Pasadena, USA

5: Also at CERN, European Organization for Nuclear Research, Geneva, Switzerland

6: Also at Laboratoire Leprince-Ringuet, Ecole Polytechnique, IN2P3-CNRS, Palaiseau, France

7: Also at Suez Canal University, Suez, Egypt

8: Also at Zewail City of Science and Technology, Zewail, Egypt

9: Also at Cairo University, Cairo, Egypt

10: Also at Fayoum University, El-Fayoum, Egypt

11: Also at British University, Cairo, Egypt

12: Now at Ain Shams University, Cairo, Egypt

13: Also at Soltan Institute for Nuclear Studies, Warsaw, Poland

14: Also at Université de Haute-Alsace, Mulhouse, France

15: Now at Joint Institute for Nuclear Research, Dubna, Russia

16: Also at Moscow State University, Moscow, Russia

17: Also at Brandenburg University of Technology, Cottbus, Germany

18: Also at Institute of Nuclear Research ATOMKI, Debrecen, Hungary

19: Also at Eötvös Loránd University, Budapest, Hungary

20: Also at Tata Institute of Fundamental Research - HECR, Mumbai, India

21: Also at University of Visva-Bharati, Santiniketan, India

22: Also at Sharif University of Technology, Tehran, Iran

23: Also at Isfahan University of Technology, Isfahan, Iran

24: Also at Plasma Physics Research Center, Science and Research Branch, Islamic Azad University, Teheran, Iran 
25: Also at Facoltà Ingegneria Università di Roma, Roma, Italy

26: Also at Università della Basilicata, Potenza, Italy

27: Also at Università degli Studi Guglielmo Marconi, Roma, Italy

28: Also at Università degli studi di Siena, Siena, Italy

29: Also at University of Bucharest, Faculty of Physics, Bucuresti-Magurele, Romania

30: Also at Faculty of Physics of University of Belgrade, Belgrade, Serbia

31: Also at University of Florida, Gainesville, USA

32: Also at University of California, Los Angeles, Los Angeles, USA

33: Also at Scuola Normale e Sezione dell' INFN, Pisa, Italy

34: Also at INFN Sezione di Roma; Università di Roma "La Sapienza", Roma, Italy

35: Also at University of Athens, Athens, Greece

36: Also at Rutherford Appleton Laboratory, Didcot, United Kingdom

37: Also at The University of Kansas, Lawrence, USA

38: Also at Paul Scherrer Institut, Villigen, Switzerland

39: Also at Institute for Theoretical and Experimental Physics, Moscow, Russia

40: Also at Gaziosmanpasa University, Tokat, Turkey

41: Also at Adiyaman University, Adiyaman, Turkey

42: Also at Izmir Institute of Technology, Izmir, Turkey

43: Also at The University of Iowa, Iowa City, USA

44: Also at Mersin University, Mersin, Turkey

45: Also at Ozyegin University, Istanbul, Turkey

46: Also at Kafkas University, Kars, Turkey

47: Also at Suleyman Demirel University, Isparta, Turkey

48: Also at Ege University, Izmir, Turkey

49: Also at School of Physics and Astronomy, University of Southampton, Southampton, United Kingdom

50: Also at INFN Sezione di Perugia; Università di Perugia, Perugia, Italy

51: Also at University of Sydney, Sydney, Australia

52: Also at Utah Valley University, Orem, USA

53: Also at Institute for Nuclear Research, Moscow, Russia

54: Also at University of Belgrade, Faculty of Physics and Vinca Institute of Nuclear Sciences, Belgrade, Serbia

55: Also at Argonne National Laboratory, Argonne, USA

56: Also at Erzincan University, Erzincan, Turkey

57: Also at KFKI Research Institute for Particle and Nuclear Physics, Budapest, Hungary

58: Also at Kyungpook National University, Daegu, Korea 